\title{
Loss of microRNA-7a2 induces hypogonadotropic hypogonadism and infertility
}

\author{
Kashan Ahmed, ${ }^{1,2}$ Mary P. LaPierre, ${ }^{1,2}$ Emanuel Gasser, ${ }^{1,2}$ Rémy Denzler, ${ }^{1,2}$ Yinjie Yang, ${ }^{1,2}$ Thomas Rülicke, ${ }^{3}$ Jukka Kero, ${ }^{4}$ \\ Mathieu Latreille, ${ }^{1,2}$ and Markus Stoffel ${ }^{1,2}$ \\ IInstitute of Molecular Health Sciences, and 2 Competence Center for Systems Physiology and Metabolic Disease, Swiss Federal Institute of Technology (ETH Zurich), Zurich, Switzerland. ${ }^{3}$ Institute of \\ Laboratory Animal Science and Biomodels Austria, Department of Biomedical Sciences, University of Veterinary Medicine Vienna, Austria. ${ }^{4}$ Departments of Pediatrics and Physiology, Institute of \\ Biomedicine, University of Turku, and Turku University Hospital, Turku, Finland.
}

\begin{abstract}
MicroRNAs (miRNAs) are negative modulators of gene expression that fine-tune numerous biological processes. miRNA loss-of-function rarely results in highly penetrant phenotypes, but rather, influences cellular responses to physiologic and pathophysiologic stresses. Here, we have reported that a single member of the evolutionarily conserved miR-7 family, miR7a2, is essential for normal pituitary development and hypothalamic-pituitary-gonadal (HPC) function in adulthood. Genetic deletion of mir-7a2 causes infertility, with low levels of gonadotropic and sex steroid hormones, small testes or ovaries, impaired spermatogenesis, and lack of ovulation in male and female mice, respectively. We found that miR-7a2 is highly expressed in the pituitary, where it suppresses golgi glycoprotein 1 (CLC1) expression and downstream bone morphogenetic protein 4 (BMP4) signaling and also reduces expression of the prostaglandin F2a receptor negative regulator (PTCFRN), an inhibitor of prostaglandin signaling and follicle-stimulating hormone (FSH) and luteinizing hormone (LH) secretion. Our results reveal that miR-7a2 critically regulates sexual maturation and reproductive function by interconnecting miR-7 genomic circuits that regulate FSH and LH synthesis and secretion through their effects on pituitary prostaglandin and BMP4 signaling.
\end{abstract}

\section{Introduction}

Sexual maturation and reproduction are regulated by the hypothalamus, pituitary, and gonads. These endocrine organs form an integrated system known as the hypothalamic-pituitary-gonadal (HPG) axis, which is responsible for the adequate secretion of male and female sex hormones (1). The endocrine components of the reproductive system are hierarchically organized and integrated in a classical endocrine feedback loop. The HPG axis is active during development and shortly after birth, silenced during childhood by inhibitory neurotransmitters, and reawakened at the onset of puberty by pulsatile secretion of gonadotropin-releasing hormone $(\mathrm{GnRH})$, a decapeptide that is synthesized by neurons located in the mediobasal hypothalamus $(2,3)$. During development, GnRH neurons originate from the olfactory placode of the olfactory system and migrate to the hypothalamus, where they control reproduction by secreting GnRH into a capillary network that transmits $\mathrm{GnRH}$ to the anterior pituitary to stimulate secretion of follicle-stimulating hormone (FSH, encoded by Fshb) and luteinizing hormone (LH, encoded by $L h b$ ). Secreted GnRH acts via the GnRH receptor, which is expressed on gonadotropic cells in the anterior pituitary gland. This action regulates synthesis and release of both gonadotropins, $\mathrm{LH}$ and $\mathrm{FSH}$, that control gonadal maturation and adult reproductive

\section{Related Commentary: p. 796}

Conflict of interest: M. Stoffel is a member of the scientific advisory board of Regulus Therapeutics.

Submitted: August 10, 2016; Accepted: December 15, 2016

Reference information: J Clin Invest. 2017;127(3):1061-1074

https://doi.org/10.1172/JCI90031. physiology via stimulation of sex steroid synthesis (1). In males, FSH stimulates proliferation of immature Sertoli cells and spermatogonia, whereas LH stimulates Leydig cells to produce testosterone (4). In females, LH triggers ovulation, promotes development of the corpus luteum, and stimulates theca cells to produce androgens, whereas FSH stimulates recruitment of secondary ovarian follicles and the secretion of estradiol from granulosa cells, thereby promoting follicular maturation, which ultimately leads to ovulation (5).

Lack of activation of the HPG axis leads to hypogonadotropic hypogonadism, which is defined as low levels of the sex steroids testosterone or estrogen (hypogonadism) in male or female patients, respectively, and inappropriately low levels of gonadotropins FSH and LH (6). An increasing number of genes have been implicated in the molecular pathogenesis of congenital isolated hypogonadotropic hypogonadism (IHH), underlining the genetic and clinical heterogeneity and complexity of this condition (7-9). Genetic causes of GnRH and gonadotropin deficiency can be grouped according to the nature of their pathogenic mechanisms, which comprise defects in neuropeptides and proteins involved in the development and migration of $\mathrm{GnRH}$ neurons (caused by mutations in KAL1, FGF8/FGFR1, PROK2/ PROKR2, CHD7, HS6ST1, WDR11, FEZF1, NSMF and SEMA3A) in genes controlling GnRH secretion and action (GNRH1, GPR54 [also known as KISS1], TAC3/TACR3, LEP/LEPR, and GNRHR, respectively) and genes participating in synthesis of $\mathrm{LH}$ or FSH $\beta$ subunits (7-9). Interestingly, homozygous inactivating mutations in the genes encoding the ubiquitin E3 ligase RNF216 and the deubiquitinase OTUD4 have recently been identified by wholeexome sequencing in patients exhibiting a syndrome of hypogonadotropic hypogonadism, progressive ataxia, and dementia, 
A

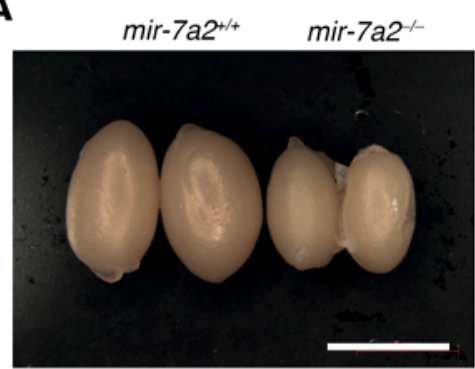

D

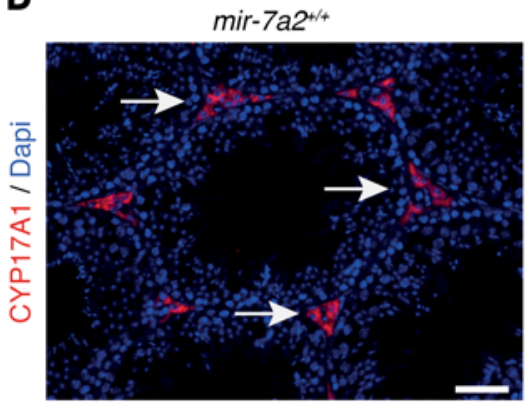

$\mathbf{F}$
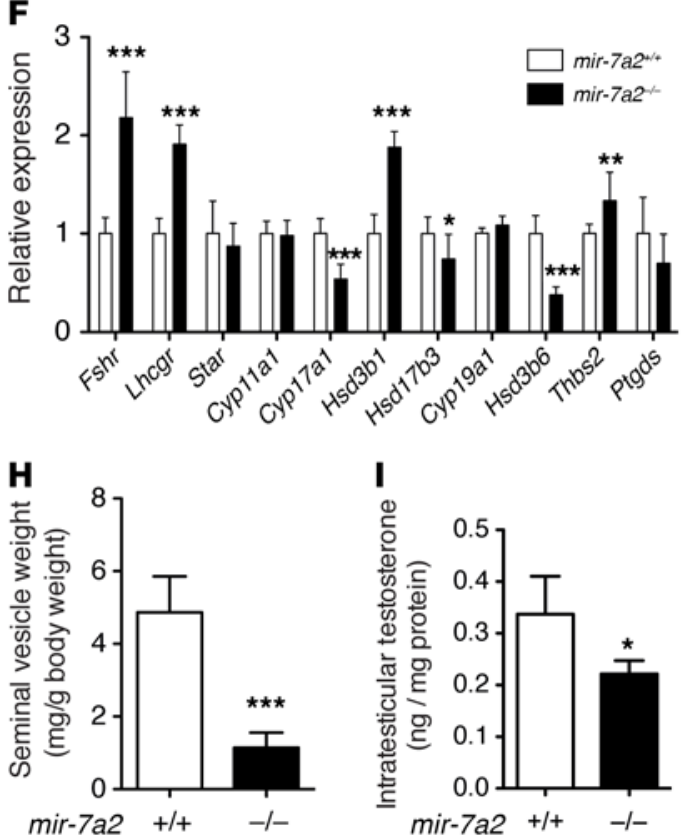

B

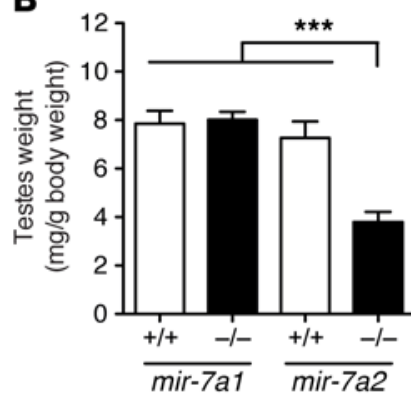

C

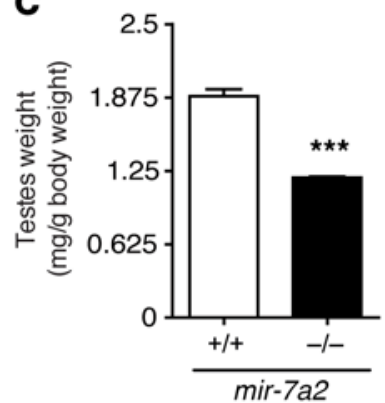

E

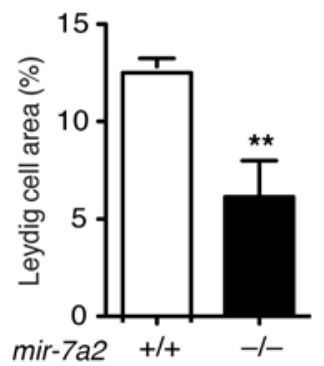

mir-7a2
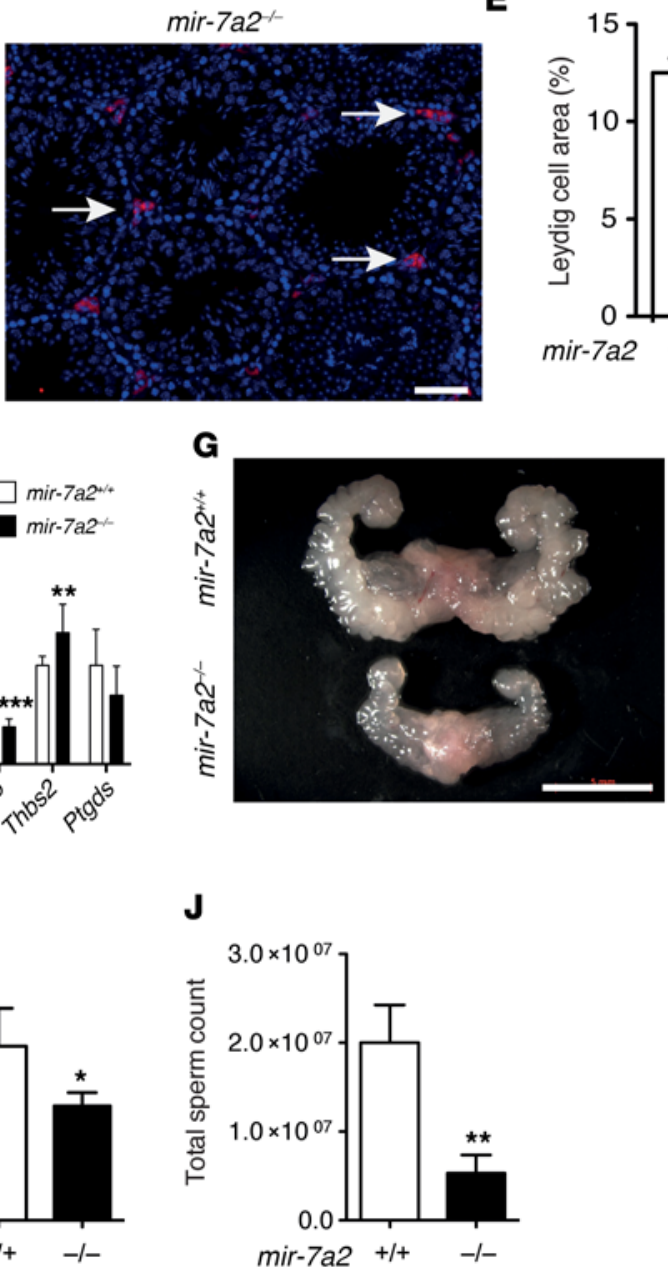

Figure 1. Ablation of mir-7a2 leads to male hypogonadism. (A-C) Representative images of mir-7a2 WT (+/+) control and KO (-/-) testes (A) and quantification of testes weights (B) of mir-7a1 K0, mir-7a2 K0, or respective WT controls (mir-7a1 control, $n=7 ;$ mir-7a1 K0, $n=6 ;$ mir-7a2 control, $n=14 ;$ mir-7a2 KO, $n=9$ ) at 6 to 8 weeks of age or (C) at 14 days (mir-7a2 control, $n=4$; mir-7a2 KO, $n=2$ ). Scale bar: $5 \mathrm{~mm}$. (D and $\mathbf{E}$ ) Immunohistological images (D) and quantification (E) of CYP17A1-positive Leydig cells (WT, mir-7a2 KO, $n=3$ ). Scale bars: $50 \mu \mathrm{m}$. Arrows show Leydig cells. (F) Relative expression levels of steroidogenic genes in testes of mir-7a2 KO or control mice (WT, $n=9$; mir-7a2 KO, $n=7$ ). ( $\mathbf{G}$ and $\mathbf{H}$ ) Representative images of seminal vesicles (G) and quantification of weights (H) of control (upper) or mir-7a2 KO (lower) mice (WT, $n=6$; mir-7a2 KO, $n=4$ ). Scale bar: $5 \mathrm{~mm}$. (I) Intratesticular testosterone levels normalized per total protein content in mir-7a2 KO or control mice (WT, $n=5$; mir-7a2 KO, $n=3$ ). (J) Total sperm count of the cauda epididymidis of 12 -week-old mir-7a2 KO or control mice (WT, $n=5$; mir-7a2 KO, $n=3$ ). All data are represented as mean $\pm \mathrm{SD}$. ${ }^{*} P<0.05 ;{ }^{*} P<0.01 ;{ }^{* *} P<0.001$, ANOVA (B); $t$ test $(\mathbf{C}, \mathbf{E}, \mathbf{F}, \mathbf{H}, \mathbf{I}, \mathbf{J})$.

thereby linking disordered ubiquitination to neurodegeneration and reproductive dysfunction (10). Despite these recent advances, the genetic basis of many cases of congenital IHH remains unknown, as these mutations account for approximately $50 \%$ of all IHH cases (11). Therefore, discovering additional gene mutations will continue to advance our understanding of this syndrome.
MicroRNAs (miRNAs) are small noncoding RNAs that act as negative regulators of gene expression by binding to miRNAresponse elements (MREs) located primarily in 3' UTRs of mRNA targets to regulate their stability and translation (12). Mammalian genomes have more than 500 miRNA genes, with miRNAs from individual gene families often targeting hundreds of different 
mRNAs $(13,14)$. With more than half of all human mRNAs estimated to be conserved miRNA targets, miRNAs are thought to have widespread effects on gene regulation. Many miRNA knockout models in mice show no apparent defect under normal conditions; however, they frequently exhibit severe miRNA-dependent phenotypes when specific stresses are applied (15). Moreover, the importance of individual miRNAs in normal physiology and disease has been established by the discovery of mutations in miRNAs and their targets (16-18). Recently, the collective role of miRNAs in the HPG axis was demonstrated by genetic ablation of Dicer1 in GnRH neurons (19) and gonadotrophs (20), resulting in hypogonadism and infertility. Furthermore, miR-200b and miR-429, both members of the miR-200 family, as well as their target, ZEB1, are required for female ovulation and reproduction in mice (21).

The miR-7 family is evolutionarily highly conserved and considered a prototypical neuroendocrine miRNA, being expressed at high levels in neurons and neuroendocrine organs, most notably the endocrine pancreas, pituitary, and adrenal glands $(14,22$, 23). Mature miR-7 harbors a conserved seed sequence that is processed from 3 precursors - miR-7a1, miR-7a2, and miR-7b - that are transcribed from unlinked loci on murine chromosomes 13, 7, and 17, respectively. A novel mechanism of miR-7 regulation was recently described in neuronal cells through the identification of a brain-enriched circular RNA (known as antisense to the cerebellar degeneration-related protein 1 transcript [Cdr1as]) comprising several dozens of conserved miR-7-binding sites counteracting repression by this miRNA $(24,25)$. Functionally, miR-7 has been implicated in organ differentiation and development (26) as well as in mammalian neurodegenerative diseases and cancer $(27,28)$. Mouse genetic studies so far have revealed a role in insulin secretion and differentiation of pancreatic $\beta$ cells (22).

In this study, we describe the unexpected finding of a hypogonadotropic hypogonadism phenotype in male and female mice as a result of the single mir-7a2 deletion. We identify miR-7a 2 as a positive regulator of FSH and LH synthesis by controlling a network involving bone morphogenetic protein 4 (BMP4) and prostaglandin signaling. Our data reveal the indispensability of a single miRNA for normal pituitary gonadotroph function, raising the question of whether genetic or acquired defects leading to altered miR-7 levels may be responsible for puberty and infertility disorders of pituitary origin.

\section{Results}

Genetic ablation of mir-7a2, but not mir-7a1 or mir-7b, leads to hypogonadism and infertility in mice. To investigate the physiological role of miR-7, we generated mice constitutively lacking mir-7a1 (referred to as mir-7a1 KO), mir-7a2 (referred to as mir-7a2 KO), or mir-7b (referred to as mir-7b KO) (Supplemental Figure 1, A-D; supplemental material available online with this article; https:// doi.org/10.1172/JCI90031DS1). Heterozygous breedings of all 3 mouse strains were viable, had unaltered body weight and body length, and were born at normal Mendelian ratios (Supplemental Figure 1, E-H). Interestingly, homozygous mir-7a2 KO male and female mice were infertile, while homozygous mir-7a1 KO or mir$7 b$ KO mice remained fertile, with unaltered reproductive rates (Supplemental Table 1).

Homozygous mir-7a2 KO male mice failed to undergo sexual maturation and exhibited microphallus and hypogonadism with approximately $50 \%$ reduced testes weights at pre- and pubertal ages ( 2 and 6 to 8 weeks old, respectively) (Figure 1, A-C). The body weights and lengths of mir-7a2 KO mice were comparable with those of WT or mir-7a1 KO animals (Supplemental Figure 1, E and $\mathrm{F}$ ). Histological analysis of mir-7a2 KO testes revealed smaller but developed seminiferous tubules, with diminished numbers of spermatocytes, spermatids, and spermatozoa (Supplemental Figure $1 \mathrm{G}$ ). Immunohistochemical quantification of the Leydig cell marker CYP17A1 showed that testosterone-producing Leydig cells were reduced by more than $50 \%$ in mir-7a2 KO mice (Figure 1, D and $\mathrm{E}$ ). We analyzed expression levels of steroidogenic enzymes in testes and measured an approximately $50 \%$ reduction of $C y p 17 a 1$ and Hsd3b6 mRNAs, which are required for dehydroepiandrosterone and testosterone synthesis, respectively (Figure 1F). Interestingly, expression levels of the FSH receptor $(F s h r)$ and $\mathrm{LH}$ receptor (Lhcgr) were increased by 2-fold, most likely due to a compensatory response to the decreased plasma gonadotrophin levels (29). The sizes and weights of seminal vesicles, a classic target organ of testosterone, were reduced by $75 \%$ (Figure 1, G and $\mathrm{H}$ ). Concomitantly, levels of intratesticular testosterone were decreased in mir-7a2 KO mice (Figure 1I) and the total sperm count of the cauda epididymidis, where mature sperm is stored, revealed a $75 \%$ decrease in mir-7a2 KO mice as compared with WT controls (Figure 1J). Finally, the inguinal fat pad weights were increased $260 \%$ in 16-week-old mice (Supplemental Figure 1H).

Analogous to the phenotype observed in males, mir-7a2 KO females had normal body weight and length (Supplemental Figure 1, I and J), but exhibited a reduced size of estrogen hormone target organs, as evidenced by reduced ovary weights and threadlike atrophied uteri compared with WT or mir-7a1 KO mice (Figure 2, A and B). Histological analysis revealed that ovaries of mir$7 a 2 \mathrm{KO}$ mice were able to form secondary and tertiary follicles that were comparable to those of WT mice; however, they lacked late-stage antral follicles or corpora lutea, consistent with failure of folliculogenesis and ovulation (Figure 2, C and D). Expression analysis of steroidogenic genes in mir-7a2 $\mathrm{KO}$ ovaries revealed a drastic decrease of Cyp19a1 mRNA, also known as aromatase, the key enzyme in estradiol biosynthesis (Figure 2E). Consequently, plasma levels of estradiol were reduced by $35 \%$ in mir-7a2 KO female mice (Figure 2F). Furthermore, as in male mice, gonadal fat pad weights were increased and dual energy $x$-ray absorptiometry (DEXA) measurements of fat and lean body mass revealed increased fat and decreased lean mass in 16-week-old mir-7a2 KO mice compared with WT littermate mice (Supplemental Figure 1, $\mathrm{K}-\mathrm{M})$, consistent with the hypogonadal-induced obesity observed in mice and humans $(30,31)$. Together, these data illustrate that loss of mir-7a2, but not mir-7a1 or mir-7b, is sufficient to induce hypogonadism and infertility in mice.

miR-7a2 is the predominant miR-7 family member and is enriched in the pituitary. Based on the increased testicular expression of Fshr and Lhcgr, which suggests decreased plasma levels of the pituitary hormones FSH and LH, we hypothesized that the gonadal dysfunction and infertility observed in male and female mir$7 a 2 \mathrm{KO}$ mice resulted from impaired pituitary function. First, we systematically analyzed expression levels of miR-7a and miR$7 \mathrm{~b}$ by absolute quantification in tissues of the hypothalamicpituitary-adrenal/gonadal axis. We found that miR-7a and miR-7b 
A

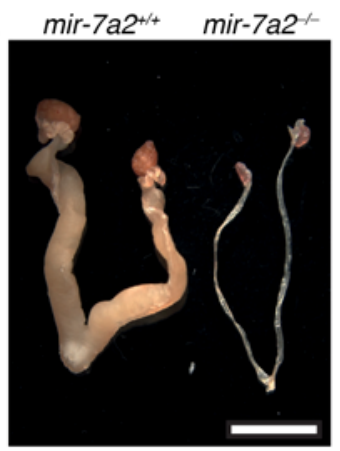

B

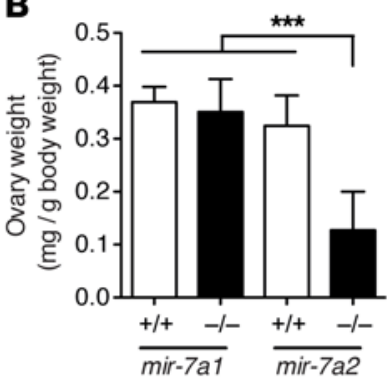

C

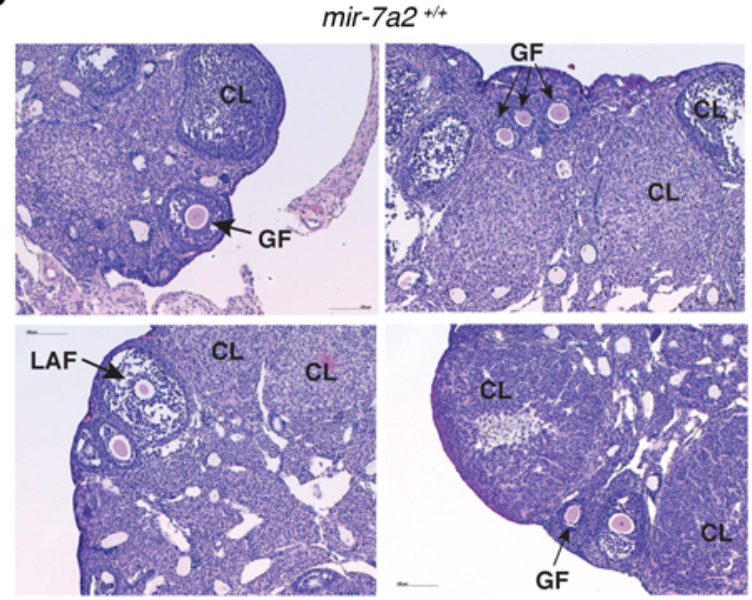

D

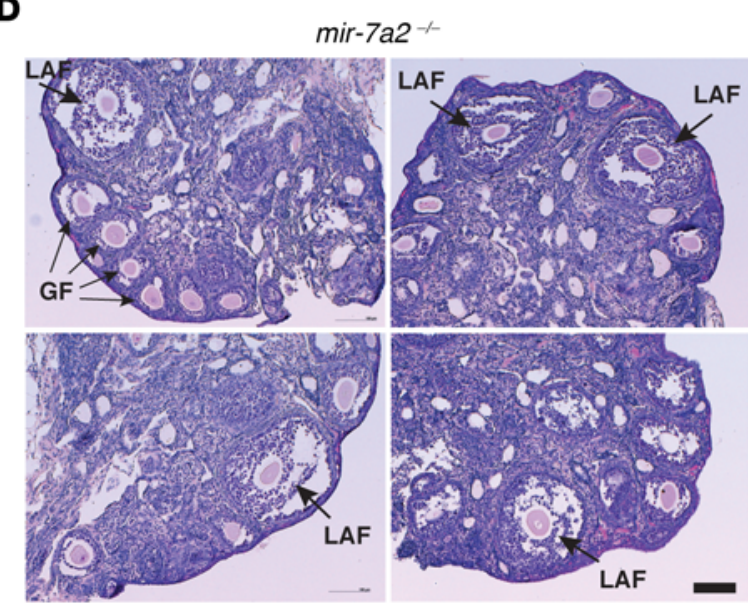

E

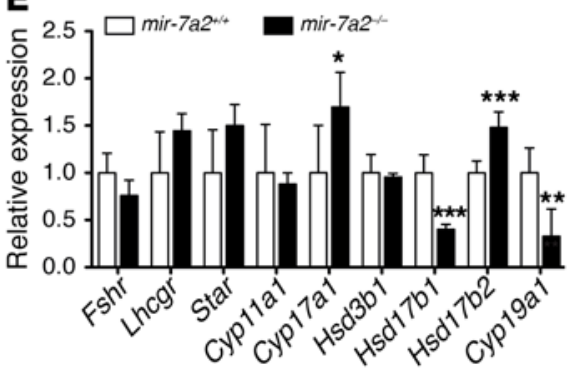

$\mathbf{F}$

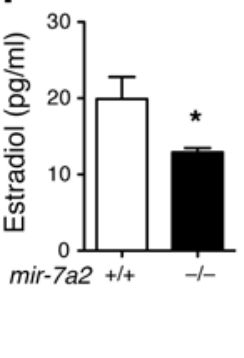

Figure 2. Ablation of mir-7a2 leads to female hypogonadism and anovulation. (A and $\mathbf{B}$ ) Representative images of ovaries and uteri (A) and quantification of ovary weights (B) of mir-7a1 KO, mir-7a2 KO, or respective control mice (mir-7a1 control, $n=9$; mir-7a1 KO, $n=10$; mir-7a2 control, $n=7$; mir-7a2 KO, $n=4$ ). Scale bar: $5 \mathrm{~mm}$. (C and D) Histological examination using H\&E staining of ovaries of WT control (C) or mir-7a2 KO (D) mice $(n=4)$. CL, corpus luteum; LAF, large antral follicle; GF, growing follicle. Shown are representative images of 4 mice per genotype. Scale bar: $100 \mu \mathrm{m}$. (E) Relative expression levels of ovarian steroidogenic genes in mir-7a2 KO or control mice (WT, $n=6$; mir-7a2 KO, $n=4$ ). (F) Plasma estradiol levels of 8-week-old mir-7a2 KO or control mice (WT, $n=12$; mir-7a2 KO, $n=11)$. All data are represented as mean \pm SD. ${ }^{*} P<0.05$; ${ }^{* *} P<0.01 ;{ }^{* *} P<0.001$, ANOVA (B); $t$ test $(\mathbf{E}, \mathbf{F})$

were enriched in pituitary as compared with hypothalamus (10fold less), adrenal gland (50-fold less), or gonads (up to 500-fold less) and that miR-7a was 2.5-fold more abundant than miR-7b in pituitary (Supplemental Figure 2, A and B). Given the low expression of miR-7a in male and female gonads, it is unlikely that miR7 a would repress target genes and exert a biological function in testis and ovary (32).

As pre-miR-7a1 and pre-miR-7a2 are processed into an identical mature miR-7a sequence, the TaqMan probe of the miRNA assay cannot distinguish between the 2 miRNA gene products and thus measures combined miR-7a1 and miR-7a2 levels. To assess the contribution of miR-7a1 and miR-7a2 to the total pool of miR-7a in the pituitary, we analyzed expression levels of miR$7 \mathrm{a}$ in mice lacking either mir-7a1 or mir-7a2. Interestingly, total miR-7a expression was reduced by approximately $80 \%$ in mir-7a2deficient mice, while miR-7a levels appeared to be unchanged in mice lacking mir-7a1 when compared with WT littermate animals (Supplemental Figure 2, C-F). Expression of miR-7b was unchanged in mir-7a1-and mir-7a2-deficient mice compared with WT animals, thereby confirming the specificity of the TaqMan assay.

In conclusion, miR-7a2 is highly enriched in pituitary compared with other neuroendocrine tissues; furthermore, it accounts for the large majority of total pituitary miR-7 copies, and miR-7a1 and miR-7b cannot compensate for loss of miR-7a2 expression.

miR-7a2 ablation causes hypopituitarism and hypogonadotropic hypogonadism. To test whether the hypogonadism observed in mir-7a2 KO mice was caused by altered gene expression of essential pituitary genes, we performed RNA sequencing (RNASeq) of pituitaries from 6-week-old WT and mir-7a2 KO mice. We measured a dramatic decrease of gonadotropin expression with a reduction of $F s h b$ mRNA by $86 \%\left(F D R=5.94 \times 10^{-52}\right)$ and $L h b$ mRNA by $78 \%\left(\mathrm{FDR}=1.26 \times 10^{-82}\right)$ in mir-7a2-deficient pituitaries that was already apparent in 2-week-old mice (Figure 3A and Supplemental Figure 3A). In addition, expression of the glycoprotein hormone $\alpha$ subunit $(C g a)$ and prolactin $(P r l)$ mRNAs was reduced, while the expression of the GnRH receptor (Gnrhr) and other hormones of the anterior pituitary, including thyroid stimulating hormone $\beta$ (Tshb), growth hormone $(G h)$, and pro-opiomelanocortin $-\alpha$ (Pomc), remained unchanged. Since lack of Prl does not lead to hypogonadism (33), we focused our investigation on the characterization of gonadotroph cells. Immunohistochemical analysis of pituitary sections revealed reduced numbers of FSH-expressing $(-66 \%)$ and LH-expressing $(-60 \%)$ cells in mir-7a2 KO mice (Figure $3, \mathrm{~B}-\mathrm{E})$. We also observed that pituitary weights were reduced 
A

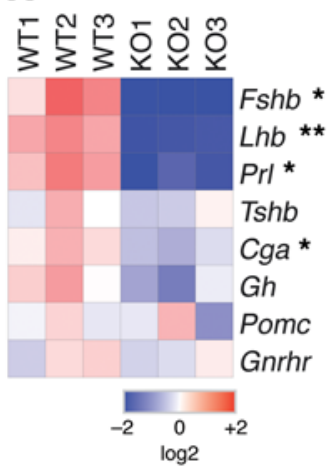

D

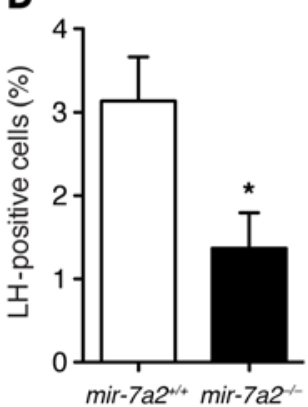

H

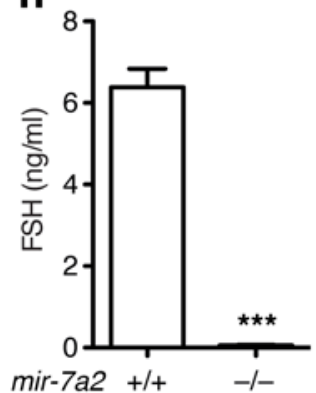

B
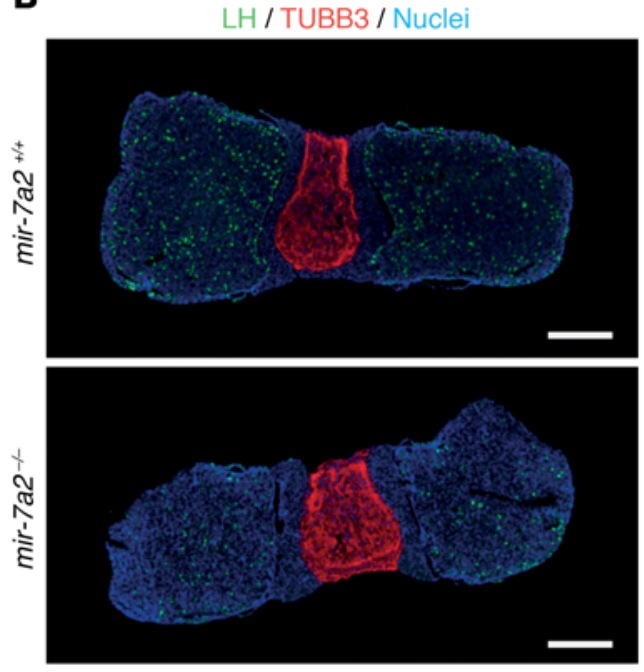

$\mathbf{F}$

E
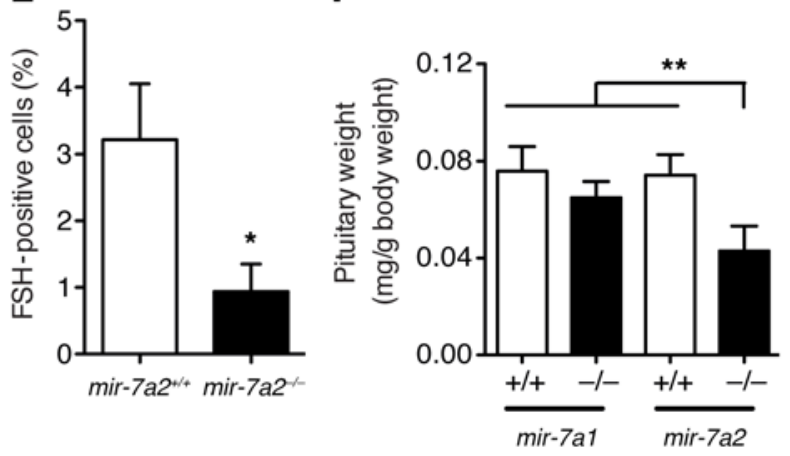

J
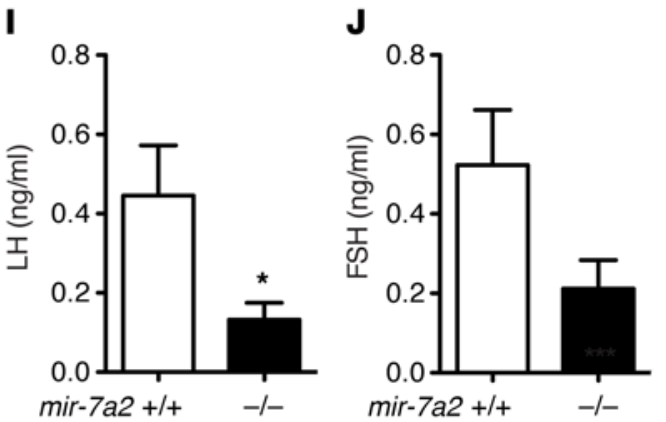

C
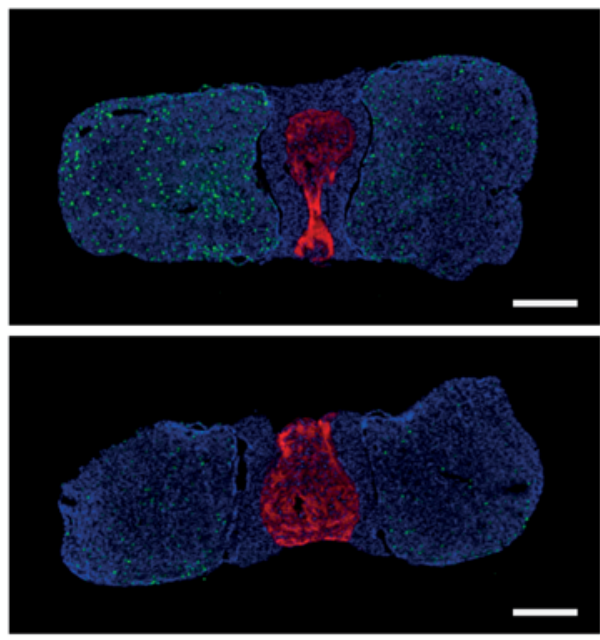

G
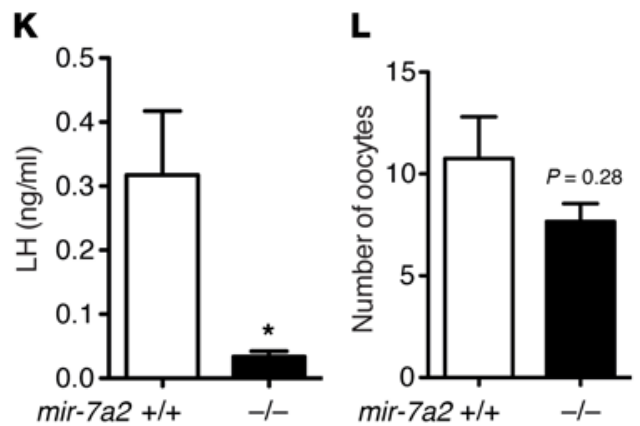

Figure 3. Constitutive genetic ablation of mir-7a2 causes hypogonadotropic hypogonadism. (A) Expression of pituitary hormones in male mir-7a2 KO or control mice shown as heat map analysis from RNA-Seq (WT, mir-7a2 KO, $n=3$ ). (B and C) Representative immunohistological images of pituitary sections $(n=3)$ stained for LH (B) or FSH (C) of control (upper images) or mir-7a2 KO mice (lower images). Scale bars: $200 \mu \mathrm{m}$. (D and E) Quantification of LH-positive (D) or FSH-positive (E) cells in pituitary of mir-7a2 KO or control mice (WT, mir-7a2 KO, $n=3$ ). (F and G) Pituitary weights of male (F) or female (G) mir-7a1 KO, mir-7a2 KO, or respective control mice (males, mir-7a1 control, mir-7a1 KO, $n=4$; mir-7a2 control, mir-7a2 K0, $n=8 ;$ mir-7a1 control, $n=8 ;$ mir-7a1 K0, $n=10$; mir-7a2 control, $n=6$; mir-7a2 KO, $n=7$ ). ( $\mathbf{H}$ and I) Plasma levels of FSH (H) or LH (I) in male mir-7a2 KO or control mice (WT, mir-7a2 KO, $n=7)$. (J and K) Plasma levels of FSH (J) or LH (K) in female mir-7a2 KO or control mice (WT, mir-7a2 KO, $n=4$ ). (L) Number of oocytes collected after superovulation test in 5-week-old mir-7a2 KO or control mice (WT, $n=4$; mir-7a2 $\mathrm{KO}, n=3$ ). All data are represented as mean \pm SD except in $(\mathbf{H}$, $\mathbf{K})$, where data are represented as \pm SEM. ${ }^{*} P<0.05 ;{ }^{* *} P<0.01 ;{ }^{* * *} P<0.001$, ANOVA (F, G); $t$ test (D, E, H, I, J, K).

in both male and female mir-7a2 KO mice (Figure 3, F and G). Importantly, the observed alterations of gene expression resulted in strongly decreased plasma concentrations of FSH and LH in male and female mir-7a2 KO mice (Figure 3, H-K). Furthermore, a GnRH stimulation test with buserelin revealed an increase of plasma FSH and LH levels in WT, but not mir-7a2 KO mice, further confirming that the response of gonadotropic cells to GnRH is impaired and that the defect in mir-7a2 $\mathrm{KO}$ mice is primarily at the level of the pituitary (Supplemental Figure 3, B and C). We then tested whether the hypopituitarism observed in mir-7a2 KO mice was responsible for the impaired gonadal function and anovulation by subjecting female mice to a standard superovulation treatment using pregnant mare serum. Ovulation was induced in hormone-treated mir-7a2 $\mathrm{KO}$ mice, indicating that ovaries were 
A

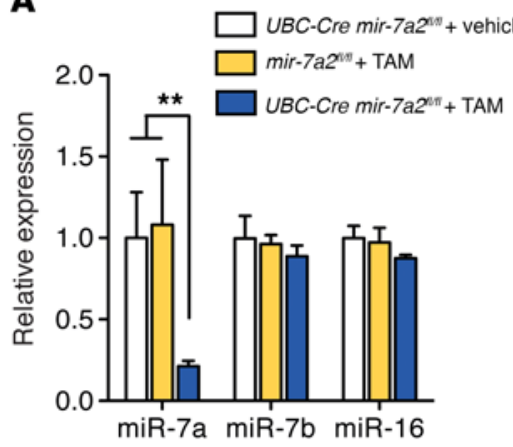

B

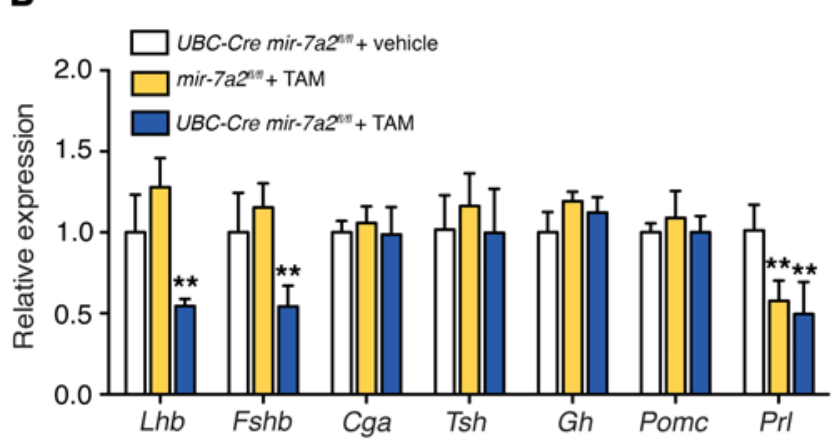

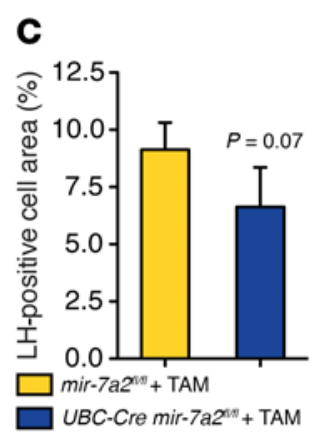

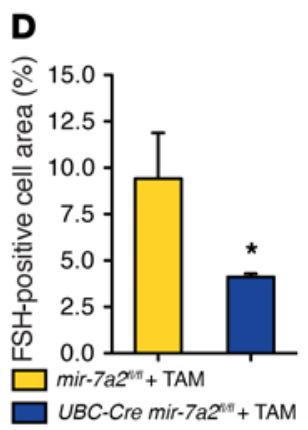

E

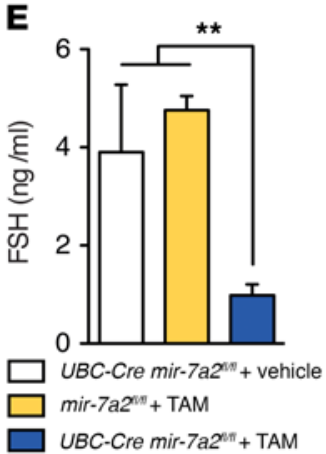

$\mathbf{F}$

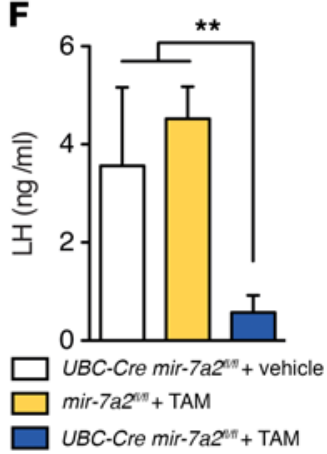

G

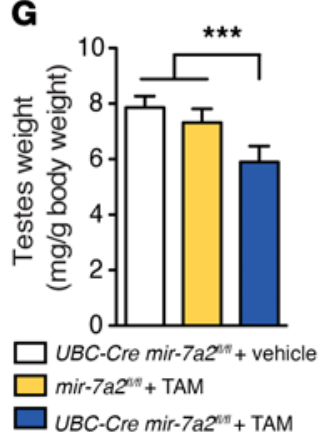

H

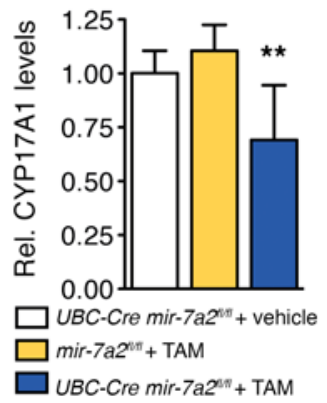

Figure 4. Acute ablation of mir-7a2 in adult mice recapitulates hypogonadotropic hypogonadism. (A) TaqMan assays showing relative expression levels of miR-7a2, miR-7b, or miR-16 in pituitary of male UBC-Cre $\times$ mir-7a fl/ff or Cre-negative mir-7a $2^{f / f f l}$ mice 10 weeks after a 5-day treatment with tamoxifen

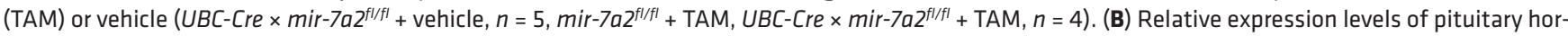

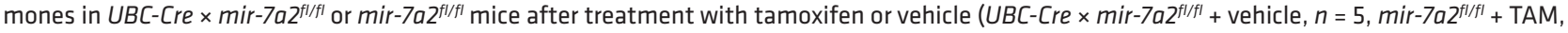
UBC-Cre $\times$ mir-7a2flox + TAM, $n=4$ ). (C and D) Immunohistological quantification of LH-positive (C) or FSH-positive (D) cells in pituitary sections of

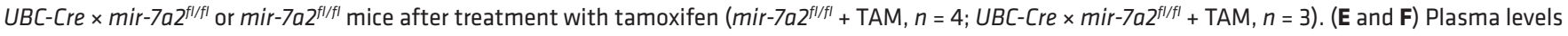
of FSH (E) or LH (F) of male UBC-Cre $\times$ mir-7a $2^{f / f l}$ or mir-7a $2^{f / f l}$ mice after treatment with tamoxifen or vehicle (FSH, UBC-Cre $\times$ mir-7a2 $2^{f / f l}+$ vehicle, $n=5$;

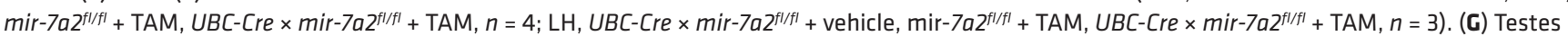

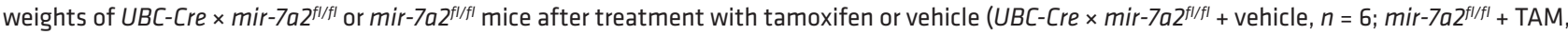

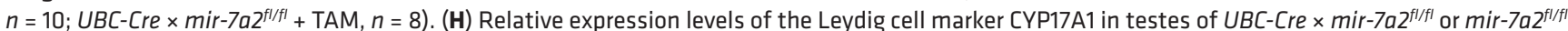

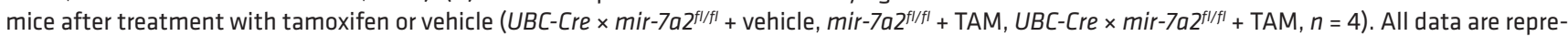
sented as mean $\pm \mathrm{SD}$. ${ }^{*} P<0.05 ;{ }^{* *} P<0.01 ;{ }^{* *} P<0.001$, ANOVA (A, B, E, F, G, H); $t$ test (C and $\left.\mathbf{D}\right)$.

principally functional and that hypophyseal-pituitary dysfunction was the primary cause for the hypogonadism in mir-7a2-ablated mice (Figure 3L). We can exclude that the relatively low expression of miR-7a in the hypothalamus affects GnRH-expressing neurons, as Gnrh mRNA levels, morphology, and expected projections of GnRH-expressing neurons were unaltered in mir-7a2 KO mice (Supplemental Figure 3, D-F). Furthermore, transcript levels of neuropeptides and genes involved in the development and migration of GnRH neurons and expression of genes con- trolling GnRH secretion and action were similar in hypothalami of mir-7a2 KO and control littermates (Supplemental Figure 3, G and $\mathrm{H}$ ). Interestingly, the neuropeptides kisspeptin 1 (Kiss1) and tachykinin 2 ( $\mathrm{Tac} 2$ ) were significantly increased in hypothalami of mir-7a2 KO mice, which corroborates findings that Kiss1 and Tac2 expression are under inhibitory modulation from circulating gonadal hormones $(34,35)$. In addition, olfactory bulbs appeared to have normal size and architecture and no difference was detected in behavioral olfactory testing of male and female mice lacking 

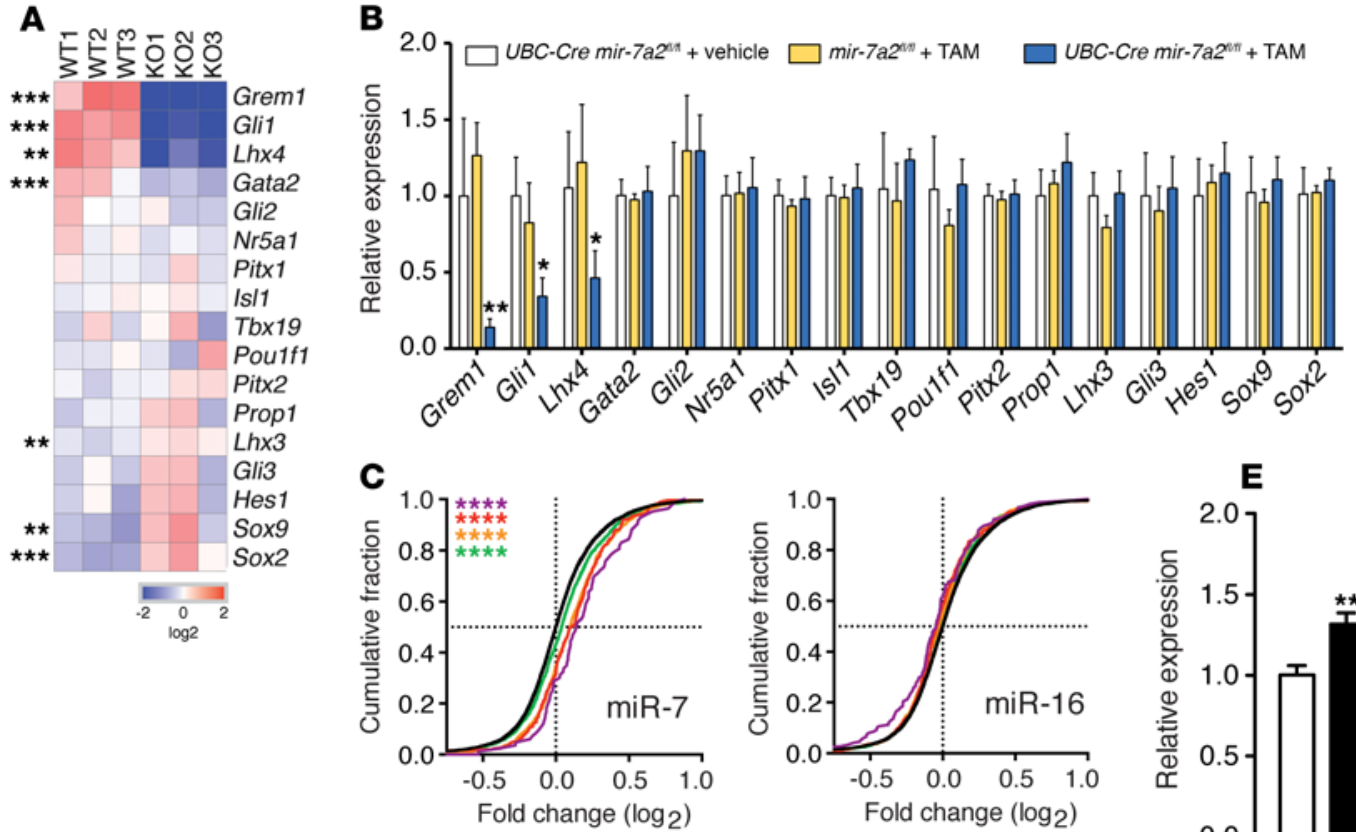

- No site 0.00 to -0.125 - CS+ -0.125 to -0.25
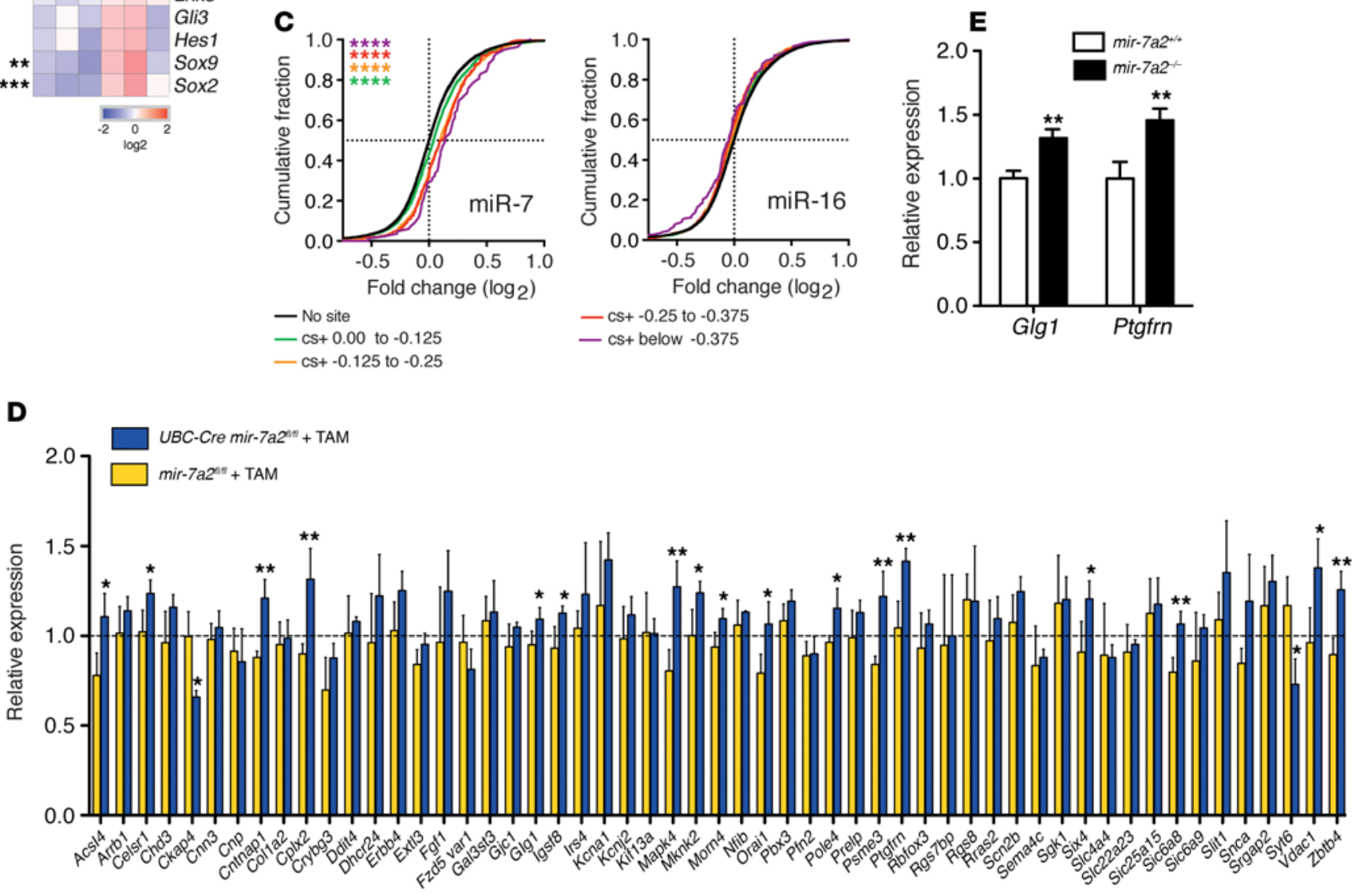

Figure 5. Pituitary gene expression analysis and identification of target genes of mir-7a2. (A) Expression of key pituitary markers in mir-7a2 KO or control mice shown as heat map analysis from RNA-Seq of 6-week-old male mice (WT, mir-7a2 KO, $n=3$ ). (B) Relative expression of key pituitary markers in UBC-

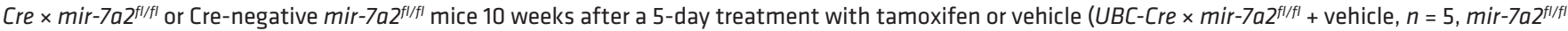
+ TAM, UBC-Cre $\times$ mir-7a $2^{f / f l}+$ TAM, $n=4$ ). (C) RNA-Seq results from pituitary of 6-week-old male mir-7a2 KO or control mice. Cumulative distributions of mRNA changes for predicted target genes of miR-7 (left)or miR-16 (right) with the indicated context ${ }^{+}$score bins (color) or for genes with no respective miRNA site (black), as scored by the context ${ }^{+}$model of TargetScan 6.2 (60). Number of genes per bin for miR-7: black, 11,884; green, 1,927; orange, 782; red 245; purple, 83; miR-16: black, 12,841; green, 656; orange, 967; red, 306; purple, 151. (WT, mir-7a2 KO, $n=3$ ). (D) Relative expression of predicted miR-7 tar-

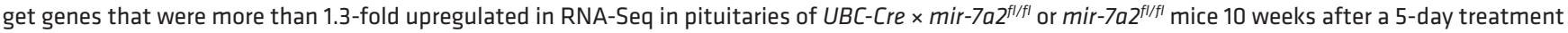

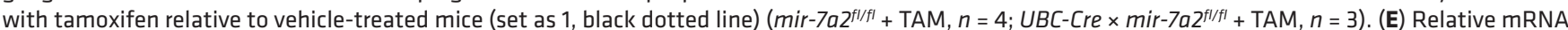

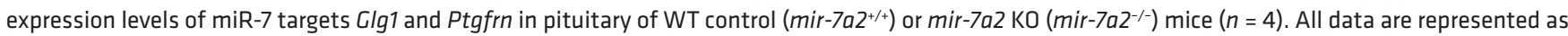
mean \pm SD. ${ }^{*} P<0.05 ;{ }^{* *} P<0.01,{ }^{* *} P<0.001,{ }^{* * *} P<0.0001$, ANOVA (B and $\left.\mathbf{C}\right) ; t$ test $(\mathbf{A}, \mathbf{D}, \mathbf{E})$.

mir-7a2 compared with littermate controls (data not shown, Supplemental Figure 3I), suggesting that the hypogonadotropic phenotype was not due to defective GnRH neuron migration from the olfactory placode into the correct hypothalamic location during development. Collectively, these data demonstrate that mir-7a2 deficiency causes pituitary dysfunction and mirrors the disease pathology of human hypogonadotropic hypogonadism.
Acute genetic ablation of miR-7a2 in adult mice recapitulates hypogonadotropic hypogonadism. To circumvent potential developmental effects caused by constitutive mir-7a2 ablation, we bred mice carrying a floxed mir-7a2 allele (referred to as mir$\left.7 a 2^{f / f f}\right)$ with transgenic mice expressing Cre-recombinase fused to a mutant form of the estrogen receptor ERT2 under the transcriptional control of the human ubiquitin $\mathrm{C}$ promoter $(U B C$ - 
A

$\square$ Ptgrin 3'UTR WT
Ptgfrn 3'UTR Mut

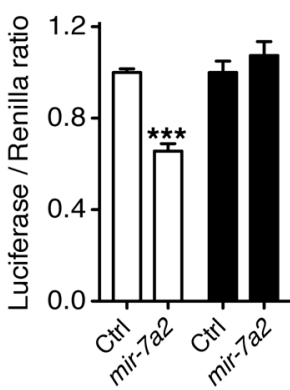

E

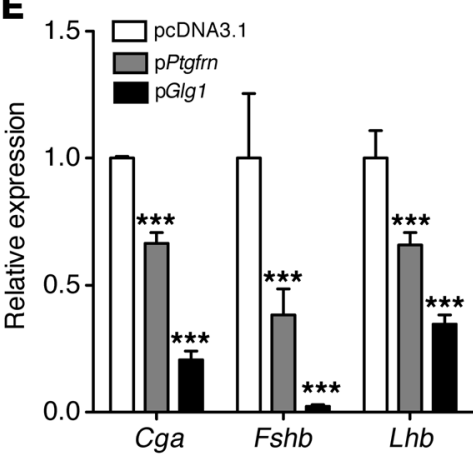

B

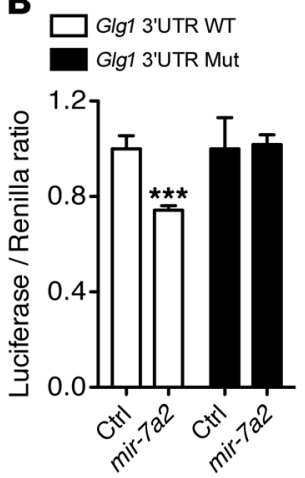

F

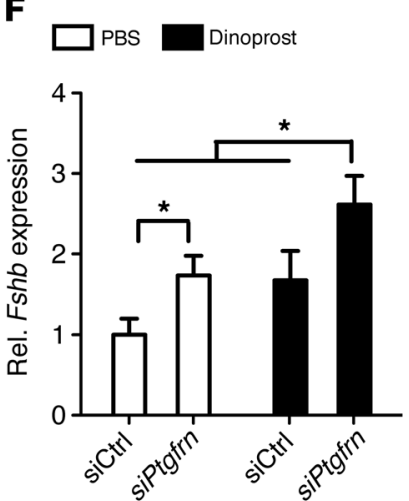

C $\square$ sictrl $\square_{\text {siPtgrn }}$

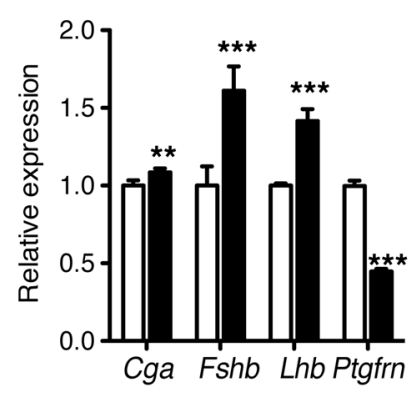

D

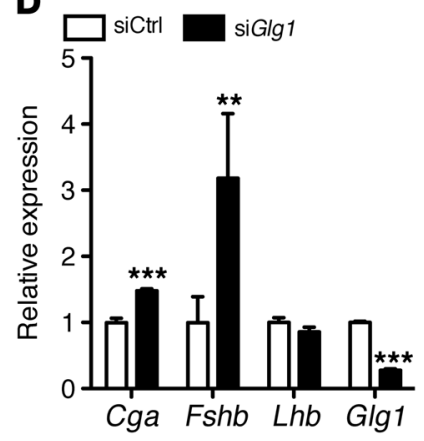

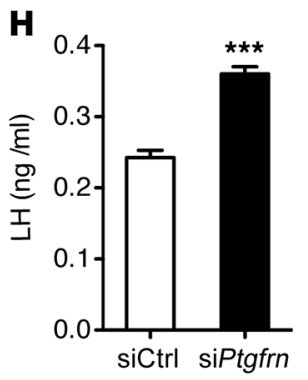

I
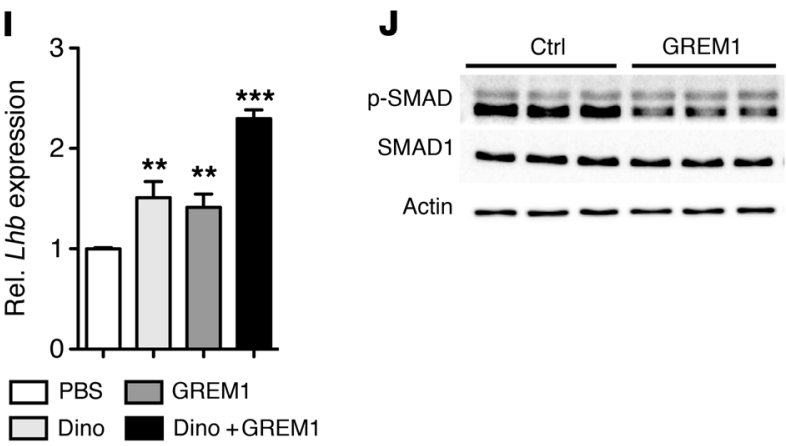

Figure 6. miR-7a2 regulates gonadotropin production through BMP and prostaglandin signaling. (A and B) Relative luciferase levels of plasmids carrying WT or mutated 3' UTRs of Ptgfrn (A) or Glg1 (B) cotransfected in LbT2 cells with or without forced expression of mir-7a2 ( $n=3$ ). (C and D) Relative expression levels of gonadotropin genes in cells transfected with siRNA against Ptgfrn (C) or Glg1 (D) ( $n=4)$. (E) Relative expression levels of gonadotropin genes, Cga, Fshb, and Lhb in cells overexpressing Ptgfrn (gray bars) or Glg1 (black bars) for 72 hours ( $n=4)$. (F and G) Relative expression levels of Fshb (F) or Lhb (G) in LbT2 cells that were transfected with siPtgfrn or siCtrl and treated with 100 nM dinoprost or PBS for 4 hours ( $n=4)$. (H) Concentration of LH in supernatants of cells 72 hours after silencing of Ptgfrn $(n=3)$. (I) Expression levels of Lhb in cells treated with dinoprost (100 nM), GREM1 ( $0.25 \mu g / m I)$, or dinoprost and GREM1 together $(n=3)$. (J) Western blot analysis of phospho-SMAD1/5/9 or total SMAD1 in lysates of cells pretreated with or without GREM1 ( $0.25 \mathrm{ug} / \mathrm{ml})$ for 1 hour, followed by 30 minutes of stimulation with BMP4 ( $50 \mathrm{ng} / \mathrm{ml})$. Shown is 1 representative experiment of 3 . All data are represented as mean $\pm \mathrm{SD}$. ${ }^{*} P<0.05 ;{ }^{* *} P<0.01 ;{ }^{* * *} P<0.001$, ANOVA (E, F, G, I); $t$ test (A, B, C, D, H).

Cre) (36) to allow tamoxifen-inducible global ablation of mir-7a2 (referred to as $U B C$-Cre $\times$ mir-7a $\left.{ }^{f / f f}\right)$. We treated 6-week-old

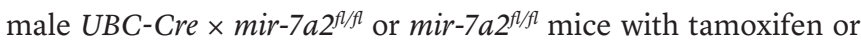
vehicle and confirmed ablation of mir-7a2 using a TaqMan miRNA assay 8 weeks after treatment (Figure 4A). While pituitary weight and body weight remained unchanged between groups (Supplemental Figure 3, J and K), a striking 50\% reduction of $F s h b$ and $L h b$ mRNA expression levels was observed in the pituitaries of tamoxifen-treated $U B C-C r e \times m i r-7 a 2^{f / f l}$ mice as com-

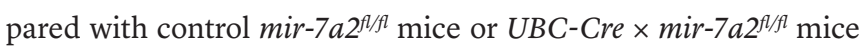

that received only vehicle (Figure 4B). Immunohistochemical quantification revealed that FSH cell area was reduced by $55 \%$ in tamoxifen-treated UBC-Cre $\times$ mir- $7 a 2^{f / f l}$ mice, while LH cell area decreased to a lesser extent, with borderline significance (Figure 4, C and D). Most notably, acute ablation of mir-7a2 in adult mice led to a drastic reduction of plasma FSH and LH levels (Figure 4, E and $\mathrm{F}$ ), and importantly, these hormones were unaltered between vehicle-treated $U B C-C r e \times \operatorname{mir}-7 a 2^{f / f l}$ and $\operatorname{mir}-7 a 2^{f l / f}$ mice that received tamoxifen (Figure $4, \mathrm{E}$ and $\mathrm{F}$ ). The decreased gonadotropin expression and secretion resulted in reduced testes weights in 


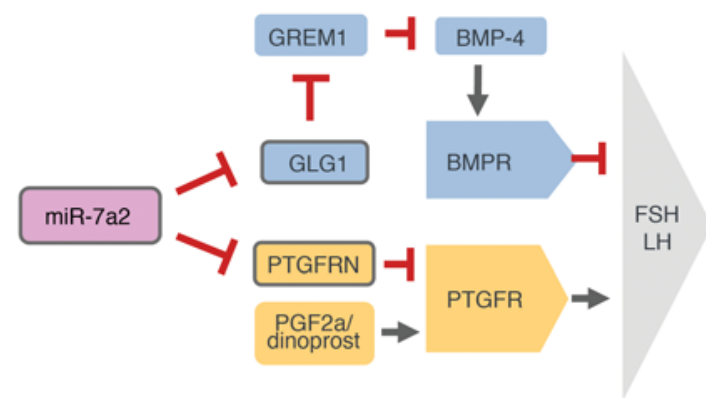

Figure 7. Model illustrating the molecular pathways by which miR-7a regulates gonadotropic hormone secretion in the pituitary.

tamoxifen-treated $U B C$-Cre $\times$ mir-7a $2^{f / f l}$ mice as compared with control mir-7a $2^{f / / f}$ mice or $U B C$-Cre $\times$ mir-7a $2^{f / f l}$ mice that received only vehicle (Figure $4 \mathrm{G}$ ). Moreover, testicular expression of the Leydig cell marker CYP17A1 was reduced by 35\% (Figure $4 \mathrm{H}$ ). Together, these data demonstrate that acute ablation of mir-7a2 in adult mice is sufficient to recapitulate hypopituitarism and hypogonadism observed in constitutive mir-7a2 KO mice.

Dysregulated pituitary gene expression and miR-7a target derepression in mir-7a2 ablated mice. To gain insights into the molecular mechanisms of miR-7a2-dependent pituitary dysfunction, we performed RNA-Seq of mir-7a2 KO and WT littermate mice and analyzed the expression of genes known to be involved in pituitary development, gonadotrope function, and/or in LH or FSH subunit gene expression. Established key factors of early pituitary function (Egr1, Gnrhr, Acvr1, Acvr1b, Acvr1c, Acvr2a, Acvr2b, Smad1, Smad2, Smad4-7, Smad9, and Foxl2) (Supplemental Figure 4A) and development (Gli2, Gli3, Nr5a1, Pitx1, Pitx2, Isl1, Tbx19, Pou1f1, Prop1, Hes1) remained unchanged between constitutive mir-7a2 KO and control mice, while Grem1, coding for gremlin 1, a secreted BMP4 antagonist (37), the zinc finger protein Gli1, and the LIM homeobox protein $4(L h x 4)$ were strongly reduced in mir-7a2 KO mice (Figure 5A). In tamoxifen-induced $U B C$-Cre $\times$ mir-7a $2^{A / A}$ mice, we confirmed that mRNA levels of Grem1, Gli1, and Lhx4 were similarly reduced, suggesting that these genes may be relevant for miR7a2-dependent effects in the pituitary (Figure 5B). Direct target genes of miRNAs were expected to be upregulated in miRNA lossof-function models. To identify potential direct targets of miR-7, we performed bioinformatic analysis and found that mRNAs carrying a miR-7 response element were preferentially upregulated in mir-7a2 KO mice (Figure 5C). No seed enrichment was observed for predicted targets of ubiquitously expressed mir-16, which is also abundant in the pituitary (Figure 5C). Interestingly, we did not measure a significant upregulation (except Pfn2 and $P r k c b$ ) of the strongest miR-7 targets previously described in pancreatic islets to regulate insulin secretion (22) (Supplemental Figure 4B), indicating a tissue-specific regulation of miR-7 target genes. Using a cut-off of 1.3 -fold upregulation, RNA-Seq revealed 55 potential direct target transcripts of miR-7. We analyzed expression of these genes in tamoxifen-induced UBC-Cre $\times$ mir-7a $2^{f / / l}$ mice and confirmed derepression of several predicted miR-7 targets (Figure 5D). Notably, Ptgfrn, the negative regulator of prostaglandin receptor F2a $(38,39)$, was as strongly upregulated as the established miR7 target Cplx2 (Ptgfrn, 1.41-fold; Cplx2, 1.32-fold) (Figure 5D). We further validated by quantitative PCR (qPCR) that the mRNA transcripts of Ptgfrn and golgi glycoprotein 1 (Glg1), a member of the cysteine-rich fibroblast growth factor receptor family (40), were increased in the pituitaries of mir-7a2 KO mice (Figure 5E). To test whether predicted mir-7 targets were repressed by increased miR-7 levels, we overexpressed miR-7a 2 using a recombinant adenovirus (Ad-miR-7a2) in 2 gonadotroph cell lines, aT3 and LbT2, and observed significant repression of several predicted miR-7 target candidates (Supplemental Figure 4, C and D). Together, these results reveal a tissue-specific regulation of direct and secondary miR-7 targets in the pituitary, with strong effects on upstream regulators of prostaglandin and BMP4 signaling.

miR-7 targets Ptgfrn and Glg1 regulate expression and secretion of pituitary gonadotropins. To investigate the role of the identified miR-7 target genes in pituitary function, we undertook an unbiased approach and silenced the 55 most regulated miR-7 targets using smart-pool siRNAs in the aT3 and LbT2 pituitary cell lines and analyzed the effect on expression levels of Fshb and Cga. We found that silencing of both Ptgfrn and Glg1 induced hormone transcription, indicating that these genes may act as negative regulators of pituitary hormone transcription (Supplemental Figure 5, A and B). The 3' UTRs of Ptgfrn and Glg1 contain 1 or $27 \mathrm{mer}$ miRNA-recognition sequences for miR-7, respectively, suggesting they are direct targets of miR-7. To confirm this, we cloned the WT or mutated 3' UTRs of Ptgfrn and Glg1 into luciferase reporter constructs, performed luciferase assays, and demonstrated that both Ptgfrn and Glg1 were direct target genes of miR-7a (Figure 6, $A$ and $B$ ). Next, we validated the findings from the siRNA screen in a separate experiment and detected a more than 1.5 -fold upregulation of Fshb when Ptgfrn or Glg1 was silenced by RNA interference (Figure 6, C and D). In addition, knockdown of Ptgfrn increased Lhb expression by 1.4-fold in LbT2 cells, while Cga was modestly regulated (Figure 6C). In contrast, overexpression of Ptgfrn or Glg1 downregulated expression of Fshb, Lhb, and Cga (Figure 6E).

PTGFRN is known to antagonize the physiological action of prostaglandin F2 $\alpha$ (PGF2 $\alpha$ ), pharmaceutically also termed dinoprost, on its cognate receptor, PTGFR $(38,39)$. Activation of PTGFR by dinoprost is coupled to stimulatory Gq/G11-type G proteins, which leads to an increase in intracellular calcium levels (41). To investigate a potential mechanistic role of PGF2 $\alpha$ signaling in gonadotroph cells, we tested whether activation of PTGFR would induce the expression of pituitary hormones in LbT2 cells following treatment with $10 \mathrm{nM}$ dinoprost. Indeed, administration of dinoprost resulted in increased expression levels of both gonadotropin genes, $F s h b$ and $L h b$, after 4 and 8 hours (Supplemental Figure 6, A and B). We then tested whether reduced activity of PTGFRN, the negative regulator of PTGFR, would further enhance the observed pharmacological effects of PGF $2 \alpha$ on pituitary hormone expression. To this end, we silenced Ptgfrn in gonadotroph cells and treated them with dinoprost for 8 hours. We found that loss of Ptgfrn further amplified the dinoprost-dependent induction of Fshb and $L h b$ (Figure 6, F and G), underscoring the specificity of a PGF2 $\alpha /$ PTGFRN/PTGFR axis to regulate hormone expression. In addition, silencing of Ptgfrn resulted in increased secretion of LH in LbT2 cells (Figure 6H).

In addition to the negative regulation of gonadotropin production by PTGFRN, BMP signaling has also been shown to interfere with hormone production in pituitary gonadotrophs $(42,43)$. Given 
that the BMP4 antagonist Grem 1 was strongly reduced in $m i R-7 a 2$ KO mice, we first tested to determine whether Grem1 overexpression would increase gonadotroph expression and detected a modest, but significant, increase in Fshb, Lhb, and Cga mRNA levels (Supplemental Figure 6C). In addition, we treated cells exogenously with recombinant GREM1 and analyzed its effect on hormone production. Interestingly, we detected an induction of hormone transcription that was comparable to that of dinoprost (Figure 6I). Most strikingly, when dinoprost and GREM1 were coadministered, we measured an additional upregulation of hormone expression (Figure 6I). Mechanistically, GREM1 inhibited BMP4-induced phosphorylation of SMAD1 in LbT2 cells when these cells were pretreated with recombinant GREM1 (Figure 6J). Moreover, we observed that GREM1 was upregulated in cells depleted in GLG1, while being decreased in cells overexpressing GLG1, indicating that the direct target GLG1 was necessary for repression of GREM1 levels (Supplemental Figure 6, D and E). Together, these data identify Ptgfrn and Glg1 as direct targets of miR-7 that negatively regulate pituitary hormone transcription and secretion through regulation of both prostaglandin- and BMP-dependent signaling pathways.

\section{Discussion}

The results presented in this study highlight the role of miR-7a2 in the regulation of pituitary function and the development of hypogonadotropic hypogonadism and infertility. Our data demonstrate that miR-7a2 is the predominant member of the miR-7 family in the pituitary and that constitutive genetic ablation of mir-7a2, but not mir-7a1, is sufficient to induce hypopituitarism and infertility. Deletion of mir-7a2 leads to decreased expression and profoundly reduced plasma levels of the pituitary gonadotropins $\mathrm{FSH}$ and $\mathrm{LH}$, resulting in hypogonadism in both male and female mir-7a2 KO mice, as indicated by decreased steroidogenic gene expression and reduced sex steroid levels and gonadal sizes. We also measured decreased expression levels of prolactin expression in constitutive and inducible mir-7a2 $\mathrm{KO}$ mice. This finding warrants further investigation and suggests that miR-7a2 either is also essential for lactotroph cell function in the pituitary or represses the function of dopaminergic neurons in the arcuate nucleus of the hypothalamus that are known to inhibit prolactin secretion (44). Interestingly, aged male and female mir-7a2 KO mice have increased fat mass, a phenotype that may be caused by a long-lasting and physiologically relevant depletion of gonadal steroid hormones (45). However, we cannot exclude that miR-7a2 also influences body weight and energy expenditure in a partially sex steroid-independent manner, for example, through neurons in the hypothalamus, where miR-7a2 expression has also been reported (46). The observed hypogonadism stems from a dysfunction of upstream components of the HPG axis, since ovarian function and ovulation are functionally rescued when pituitary hormones are replaced in $\mathrm{miR}-7 a 2$ $\mathrm{KO}$ mice in a superovulation test. This phenotype is reminiscent of the disease pathology of human hypogonadotropic hypogonadism, prompting the question of whether some cases of congential IHH of pituitary origin with as-yet-unknown genetic causes may be attributed to abnormal miR-7a2 levels. Further investigations are warranted to explore this possibility.

Cases of Kallmann's syndrome, a form of human hypogonadotropic hypogonadism, are often accompanied by a loss of smell, termed anosmia, due to defective migration of GnRH neurons from the olfactory placode into the hypothalamus during development and the subsequent lack of GnRH release to the pituitary, leading to hypopituitarism (47). Our results show that the hypogonadotropic hypogonadism in mir-7a2 KO mice is highly unlikely to be causally linked to miR-7 function in hypothalamic neurons because (a) absolute quantification of miR-7 levels demonstrates that miR-7a expression is 10-fold lower in hypothalamus as compared with pituitary and therefore, based on miR-7b levels, in a range where no phenotype and target gene regulation is expected; (b) hypothalamic GnRH expression levels are indistinguishable between mir-7a2 KO and control littermates; (c) mir-7a2 KO mice show unaltered olfaction and normal expression of hypothalamic genes that are known to be involved in hypothalamus-dependent hypopituitarism; and (d) expression of the GnRH receptor in the pituitary, which is downregulated in animal models of GnRH deficiency, is expressed at similar levels in mir-7a2 KO and WT control mice (48). In addition, miR-7 is not among the 53 miRNAs that are enriched in GnRH neurons compared with the surrounding nonGnRH cells (19), further suggesting that miR-7 is unlikely to have a specific function in these neurons.

We have previously shown that loss of miR-7a2 in pancreatic $\beta$ cells leads to increased $\beta$ cell function and enhanced glucosestimulated insulin secretion (22). Interestingly, ablation of mir-7a2 in the pituitary leads to impaired gonadotroph cell function and hormone secretion. These opposing phenotypes, elicited by a loss of the same miRNA in 2 different neuroendocrine cell types, suggest that miR-7a regulates different networks in a tissue-specific and context-dependent manner. The molecular mechanism for this observation is currently elusive, but will likely be due to different miRNA and target abundances and synergistic regulation of target genes by differentially expressed miRNAs as well as diverse occupancy of transcripts by RNA-binding proteins, which may influence binding of miRNAs to their target transcripts, thereby regulating the susceptibility of miRNA regulation.

The miR-7 family has previously been implicated in organ differentiation and development (22). Our results showing already reduced gonad sizes and dysregulated expression of FSH and $\mathrm{LH}$ as well as direct and indirect miR-7 targets in prepubertal mice indicate that a developmental defect contributes to the hypogonadotrohic phenotype observed in mice with constitutive genetic ablation of mir-7a2. However, our data obtained from temporally induced mir-7a2 ablation in postdevelopmental adult mice provide genetic evidence that impaired gonadotroph cell functions cannot solely be ascribed to a developmental defect and demonstrate a direct role of miR-7a in the maintenance of pituitary gonadotroph function in adult animals. Mechanistically, we identify the negative regulator of PGF2 $\alpha$ receptor, Ptgfrn, and Glg1 as relevant direct target genes of miR-7a that negatively regulate gonadotropin production and secretion. While only these targets could be linked to gonadotropin regulation in our pituitary cell models, we cannot rule out that other miR-7 targets also contribute to the defect in gonadotroph development and function of mir-7a2 KO mice. The physiological effects of prostaglandins on pituitary gonadotrophs have not been fully elucidated and have been in part discussed controversially. While it is accepted that prostaglandins, particularly PGE1, act on the anterior pituitary and can induce the formation of 
cyclic AMP, a direct effect on LH and FSH could not be established (49). In the case of PGF2 $\alpha$, known as dinoprost, however, existing data suggest that PGF $2 \alpha$ treatment may regulate LH stores and release (50-52). Our data now demonstrate that in pituitary gonadotroph cell lines, PGF2 $\alpha$ /dinoprost increases gonadotropin production and that the direct miR-7a target PTGFRN acts as a negative modulator in a PGF2 $\alpha /$ PTGFRN/PTGFR regulatory circuit.

In addition, we here describe the inhibitory role of the miR-7a target GLG1 in gonadotroph cell functions. While the precise physiological functions of GLG1 in pituitary hormone homeostasis are unknown, our data suggest that GLG1 negatively affects expression of the BMP antagonist GREM1. BMP4 plays a crucial role in the formation of the anterior pituitary by regulating diverse cellular responses, such as cell differentiation, migration, adhesion, and proliferation (53), but also has been described as inhibiting FSH production $(42,54)$. BMPs activate BMP receptors, which in turn recruit and phosphorylate SMAD proteins that translocate to the nucleus to repress Fshb subunit gene expression. Furthermore, BMPs can antagonize the activin-mediated effects known for stimulating FSH release (42). We propose that in miR-7a2 KO mice, the drastic reduction in GREM1 levels is partly mediated by dysinhibition of its negative regulator and miR-7 target GLG1, leading to derepression of BMP4 and, consequently, increased BMP activity, which affects pituitary development defect and impairs gonadotroph function in adult mice (Figure 7). Indeed, in this context, we demonstrate that GREM1 acts as a BMP4 antagonist to reduce phosphorylation of SMAD proteins in pituitary LbT2 cells.

In summary, our data reveal miR-7a2 as a crucial regulator of pituitary gonadotroph cell function and describe how the loss of mir-7a2 leads to hypogonadotropic hypogonadism. Mutations in the $m i R-7 a 2$ gene and within the binding sites in the $3^{\prime}$ UTRs of its most regulated targets should be considered as potential genetic causes for hypogonadotropic hypogonadism in humans. Our findings also characterize PTGFRN and GLG1 as two novel target genes of miR-7 that negatively regulate gonadotropin production. Pharmacological stimulation of miR-7 activity and/or direct inhibition of its targets PTGFRN and GLG1 may provide a basis to develop strategies to improve pituitary function in human disease.

\section{Methods}

Animal husbandry and mouse strains. All mice were on a pure C57BL/6N background. Mice were housed in a pathogen-free animal facility at the Institute of Molecular Health Sciences at ETH Zurich. The animals were maintained in a temperature- and humidity-controlled room on a 12-hour light/12-hour dark cycle (lights on from 6 am to $6 \mathrm{pm}$ ). Mice were fed a standard laboratory chow diet. Generation of mir-7a1 KO and mir-7a2 KO mice was described previously (22). UBC-Cre mice (Tg[UBC-cre/ERT2]1Ejb) were purchased from Jackson Laboratories.

Generation of mir-7b KO mice. A 15.7-kb fragment encompassing $\mathrm{mmu}-\mathrm{mir}-7 \mathrm{~b}$ was recombined into a minimal vector using homology arms-containing primers according to the protocol provided by GeneBridges (http://www.genebridges.com; primers listed in Supplemental Table 2). The mir-7b gene was juxtaposed with a loxP-containing neor cassette $97 \mathrm{bp}$ upstream of the miRNA precursor sequence. Following expression of the Cre in bacteria, the $n e o^{r}$ cassette was removed and a second Frt/lox $\mathrm{P} /$ neo $^{r}$ cassette was introduced 498 bp downstream of the miR-7b gene (Supplemental Figure 1A). Upon linearization, the targeting vector was electroporated in C5BL/6N embryonic stem cells. Upon selection with G418 (Invitrogen), clones were picked, expanded, and verified for integration by Southern blotting. Positive clones were microinjected in BALB/c blastocytes and subsequently transferred into pseudopregnant females to generate chimeric offspring. Chimeras were then bred with C57BL/6 females to produce heterozygotes, which were crossed with Flipase mice to delete the neor cassette. Mice were bred with B6.C-Tg(CMV-cre)1Cgn/j mice (Jackson Laboratory) for deleting mir-7b. Genotyping of mir-7b KO mice was performed by PCR using primers listed in Supplemental Table 2.

DEXA. DEXA measurements of fat and lean body mass were performed as whole animal scans using an animal CT-Scanner (LaTheta) at 1-mm intervals from the head to the base of the tail. Images were analyzed using the LaTheta software.

Immunostainings. Mice were perfused with $4 \%$ paraformaldehyde, and organs were postfixed overnight at $4^{\circ} \mathrm{C}$ and embedded in paraffin. Microtome sections were subjected to antigen retrieval in a pressure oven in $10 \mathrm{mM}$ sodium citrate, $\mathrm{pH} 6.0$, for 10 minutes at $95^{\circ} \mathrm{C}$. Sections were permeabilized and blocked in PBS containing 0.1\% Triton $\mathrm{X}-100,1 \%$ BSA, and $5 \%$ goat serum. Primary antibody binding was performed overnight at $4^{\circ} \mathrm{C}$, while secondary antibody incubation was carried out at room temperature for 1 hour.

For morphometric analysis of pituitaries, $5-\mu \mathrm{m}$ sections were cut and 5 sections from each pituitary were stained with anti-LH or anti-FSH antibodies and Hoechst dye to stain nuclei. Sections were scanned using a $20 \times$ objective of a Zeiss Apotome microscope. The number of FSH- or LH-positive cells compared with total cell number was determined using CellProfiler software. For morphometrical quantification of testicular Leydig cells, 4- $\mu \mathrm{m}$ paraffin sections were cut and 5 sections per testis were stained with anti-CYP17A1 antibody and Hoechst dye to stain nuclei. Sections were scanned using a $20 \times$ objective of the Panoramic 250 Slide Scanner (3D Histech). The area fractions of CYP17A1-positive cells relative to total cell area were determined using ImageJ (Fiji, NIH) software.

Western blots. Cells were lysed in RIPA buffer containing protease inhibitors (Roche) and HALT phosphatase inhibitors (Pierce). Protein concentrations were measured by the Bradford method. Proteins were separated by SDS-PAGE, transferred to nitrocellulose membranes, and blocked in 5\% milk/TBS-T or 5\% BSA/TBS-T for 1 hour. Membranes were incubated with appropriate antibodies overnight at $4^{\circ} \mathrm{C}$. Membranes were exposed to secondary antibodies for 1 hour at room temperature and developed using ECL Western Blotting Substrate.

Illumina RNA-Seq and analysis. Library preparation was as follows: the quality of the isolated RNA was determined with a Qubit (1.0) Fluorometer (Life Technologies) and a Bioanalyzer 2100 (Agilent). Only those samples with a 260/280 nm ratio between 1.8 and 2.1 and a 28S/18S ratio within 1.5 and 2.0 were further processed. The TruSeq RNA Sample Prep Kit v2 (Illumina) was used in the succeeding steps. Briefly, total RNA samples (100-1000 ng) were poly-A enriched and then reverse transcribed into double-strand cDNA. The cDNA samples were fragmented, endrepaired, and polyadenylated before ligation of TruSeq adapters containing the index sequence for multiplex sequencing. Multiplexing fragments containing TruSeq adapters on both ends were selectively enriched with PCR. The quality and quantity of the enriched libraries were validated using Qubit (1.0) Fluorometer and the Caliper GX LabChip GX (Caliper Life Sciences Inc.). The product is a smear with an average fragment size of approximately 
$260 \mathrm{bp}$. The libraries were normalized to $10 \mathrm{nM}$ in Tris- $\mathrm{Cl} 10 \mathrm{mM}, \mathrm{pH}$ 8.5, with $0.1 \%$ Tween 20.

Cluster generation and sequencing. The TruSeq PE Cluster Kit v3-cBot-HS or TruSeq SR Cluster Kit v3-cBot-HS (Illumina Inc.) was used for cluster generation using $10 \mathrm{pM}$ of pooled normalized libraries on the cBOT. Sequencing was performed on the Illumina HiSeq 2000 paired end at $2 \times 101 \mathrm{bp}$ or single-end $100 \mathrm{bp}$ using the TruSeq SBS Kit v3-HS (Illumina Inc.).

Data analysis. RNA-Seq reads were quality checked with FastQC (Babraham Bioinformatics), which computes various quality metrics for raw reads. Reads were aligned to the genome and transcriptome with TopHat v 1.3.3 (https://ccb.jhu.edu/software/tophat/manual.shtml). Before mapping, the low-quality ends of the reads were clipped ( 3 bases from the read start and 10 bases from the read end). TopHat was run with default options. The fragment length parameter was set to 100 bases, with an SD of 100 bases. Based on these alignments, the distribution of the reads across genomic features was assessed. Isoform expression was quantified with the RSEM algorithm (http://www. biomedcentral.com/1471-2105/12/323; BMC Bioinformatics) with the option for estimation of the read start position distribution turned on. For seed enrichment analysis, only genes with FPKM above 1.0 were considered. For cumulative distribution function calculations, $\log _{2}$ fold-change values were corrected for 3' UTR length biases (55).

RNA isolation and real-time pPCR. RNA was isolated using TRIzol reagent (Invitrogen) according to the manufacturer's protocol. RNA was subjected to DNaseI treatment with the DNA-free kit (Ambion) when necessary. RNA was reverse transcribed using the High Capacity cDNA Reverse Transcription Kit (Applied Biosystems). qPCR was performed with a LC480 II Lightcycler (Roche) and SYBR Fast Universal Mastermix (Kapa). Results were normalized to 36B4 mRNA levels. miRNA levels were measured using the TaqMan microRNA Assay Kit (Applied Biosystems), and the results were normalized to sno-202 levels. For absolute quantification, synthesized mature miRNAs were used (Sigma-Aldrich).

Luciferase assays. LbT2 or aT3 cells were cultured in 24-well plates and transfected with $100 \mathrm{ng}$ of pmirGLO reporters. Cells were assayed 48 hours after transfection using the Dual-Luciferase Reporter Assay System (Promega). Results were normalized to the Renilla luciferase control contained in pmirGLO and expressed relative to the average value of the controls.

GnRH stimulation test. Female nullipara mice were given an intraperitoneal injection of Buserelin (GnRH agonist, Sigma-Aldrich; 0.01 $\mu \mathrm{g} / \mu \mathrm{l}$ ) in $200 \mu \mathrm{l}$ of vehicle (PBS) or vehicle alone and blood was collected after 15 minutes for FSH and LH measurements.

Hormone measurements. Testosterone and estradiol were quantified using the ELISA Kit from Alpco following the manufacturer's instructions. For intratesticular testosterone analysis, lipid extraction was performed using $2 \times$ diethylether. Serum LH and FSH concentrations were measured using immunofluorometric assays as described previously (55). For measuring intratesticular and serum testosterone, both sera and tissue homogenates were extracted twice with $2 \mathrm{ml}$ of diethyl ether, followed by conventional RIA (56).

Olfaction behavioral test. A buried food test was conducted as previously described (57). Nine-week-old mice were fasted for 24 hours and then were allowed to acclimate in the experimental cage for $5 \mathrm{~min}$ utes prior to the test. Mice were transferred to an empty cage, while a $1.5 \mathrm{~g}$ pellet of chow was buried $1 \mathrm{~cm}$ below the bedding in the experi- mental cage. Mice were reintroduced into the experimental cage, and the elapsed time was recorded once they uncovered the chow pellet.

Recombinant adenovirus. Mouse miR-7a2-expressing vectors were generated by PCR amplification of a 250-bp fragment spanning the pre-miR-7a2 genomic sequences. Fragments were cloned at XhoI sites of pAD5 for adenovirus production (Viraquest). The miR-7a adenoviruses expressed GFP from an independent promoter, and the control adenovirus (Ad-GFP) was identical except that it lacked the miRNA. Expression was confirmed by TaqMan qPCR.

Chemical compounds. BMP4, dinoprost, and GnRH were purchased from Sigma-Aldrich. Grem1 was obtained from R\&D Systems.

Cell lines. Pituitary cell lines $\alpha$ T3 and L $\beta$ T2 were provided by P. Mellon (UCD, Davis, California, USA) $(58,59)$.

Antibodies. Antibodies recognizing mouse $\mathrm{FSHb}$ and $\mathrm{LHb}$ were obtained from A. Parlow (National Hormone and Peptide Program, Harbor-UCLA Medical Center, Torrance, California, USA). TUBB3 (catalog 4466), $\beta$-actin (catalog 4970), SMAD1 (catalog 6944), and phospho-SMAD1/5/9 (catalog 13820) were purchased from Cell Signaling Technology. CYP17A1 antibody was purchased from Santa Cruz Biotechnology Inc. (catalog sc-46081).

Primer sequences. See Supplemental Table 3.

Statistics. For all experiments, data are shown as mean \pm SD if not stated otherwise. To determine statistical significance between 2 groups, 2-tailed Student's $t$ test was used. For multiple comparisons, 1-way ANOVA followed by Tukey's post-test was used. A $P$ value of less than 0.05 was considered statistically significant. Statistical analysis was performed using GraphPad Prism 4 (GraphPad software).

Study approval. All animal experiments were approved by the Kantonale Veterinäramt Zürich, Zurich, Switzerland.

Accession codes. All raw RNA-Seq data were deposited in the European Nucleotide Archive (ENA PRJEB12612; http://www.ebi.ac.uk/ena).

\section{Author contributions}

KA designed and performed most of the experiments, analyzed and interpreted data, and wrote the manuscript. ML generated constitutive and conditional mir-7a1, mir-7a2, and mir-7b KO mice. MPL contributed to in vivo and cell culture experiments and immunohistochemistry. JK performed FSH and LH measurements. RD performed seed enrichment analysis. EG performed hypothalamus immunohistochemistry. YY contributed to cell culture experiments. TR performed blastocyst injections. MS conceived and supervised the project, analyzed and interpreted data, and wrote the manuscript. All authors read and commented on the manuscript.

\section{Acknowledgments}

We wish to thank Hasan Kabakci and Regina Kubsch for excellent technical and animal husbandry assistance and Taina Kirjonen for skillful assistance with gonadotropin measurements. We are grateful to Pamela Mellon for providing pituitary cell lines. We thank the Functional Genomics Center Zurich and the Light Microscopy and Screening Center Zurich for support. This work was supported by European Molecular Biology Organization (EMBO) long-term fellowships (to KA), the Austrian Genome Research Program GEN-AU II and III (Austromouse) (to TR), European Research Council (ERC) grant "Metabolomirs," the Starr Foundation International and the Swiss National Science Foundation, and the National Center of Competence in Research (NCCR) on RNA Biology and Disease (to MS). 
Address correspondence to: Markus Stoffel, Institute of Molecular Health Sciences, Swiss Federal Institute of Technology (ETH Zurich), Otto-Stern Weg 7, 8093 Zurich, Switzerland. Phone: 41.44.633.4560; E-mail: stoffel@biol.ethz.ch.
ML's present address is: Cellular Identity and Metabolism Group, MRC London Institute of Medical Sciences London, United Kingdom; or Institute of Clinical Sciences, Faculty of Medicine, Imperial College London, London, United Kingdom.
1. Abreu AP, Kaiser UB. Pubertal development and regulation. Lancet Diabetes Endocrinol. 2016;4(3):254-264.

2. Herbison AE. Control of puberty onset and fertility by gonadotropin-releasing hormone neurons. Nat Rev Endocrinol. 2016;12(8):452-466.

3. Leon S, Tena-Sempere M. Dissecting the roles of gonadotropin-inhibitory hormone in mammals: studies using pharmacological tools and genetically modified mouse models. Front Endocrinol (Lausanne). 2016;6:189.

4. Ramaswamy S, Weinbauer GF. Endocrine control of spermatogenesis: Role of FSH and LH/ testosterone. Spermatogenesis. 2015;4(2):e996025.

5. Christensen A, et al. Hormonal regulation of female reproduction. Horm Metab Res. 2012;44(8):587-591.

6. Brioude F, et al. Non-syndromic congenital hypogonadotropic hypogonadism: clinical presentation and genotype-phenotype relationships. Eur J Endocrinol. 2010;162(5):835-851.

7. Stamou MI, Cox KH, Crowley WF Jr. Discovering genes essential to the hypothalamic regulation of human reproduction using a human disease model: adjusting to life in the -omics Era. Endocr Rev. 2015;36(6):603-621.

8. de Roux N, Carel JC, Léger J. Congenital hypogonadotropic hypogonadism: a trait shared by several complex neurodevelopmental disorders. Endocr Dev. 2016;29:72-86.

9. Topaloglu AK, Kotan LD. Genetics of hypogonadotropic hypogonadism. Endocr Dev. 2016;29:36-49.

10. Margolin DH, et al. Ataxia, dementia, and hypogonadotropism caused by disordered ubiquitination. N Engl J Med. 2013;368(21):1992-2003.

11. Boehm U, et al. Expert consensus document: European Consensus Statement on congenital hypogonadotropic hypogonadism--pathogenesis, diagnosis and treatment. Nat Rev Endocrinol. 2015;11(9):547-564.

12. Guo H, Ingolia NT, Weissman JS, Bartel DP. Mammalian microRNAs predominantly act to decrease target mRNA levels. Nature. 2010;466(7308):835-840.

13. Friedman RC, Farh KK, Burge CB, Bartel DP. Most mammalian mRNAs are conserved targets of microRNAs. Genome Res. 2009;19(1):92-105.

14. Landgraf $\mathrm{P}$, et al. A mammalian microRNA expression atlas based on small RNA library sequencing. Cell. 2007;129(7):1401-1414.

15. Mendell JT, Olson EN. MicroRNAs in stress signaling and human disease. Cell. 2012;148(6):1172-1187.

16. de Pontual L, et al. Germline deletion of the miR17 92 cluster causes skeletal and growth defects in humans. Nat Genet. 2011;43(10):1026-1030.

17. Mencía $\mathrm{A}$, et al. Mutations in the seed region of human miR-96 are responsible for nonsyndromic progressive hearing loss. Nat Genet . 2009;41(5):609-613.

18. Dusl M, et al. A 3'-UTR mutation creates a
microRNA target site in the GFPT1 gene of patients with congenital myasthenic syndrome. Hum Mol Genet. 2015;24(12):3418-3426.

19. Messina A, et al. A microRNA switch regulates the rise in hypothalamic $\mathrm{GnRH}$ production before puberty. Nat Neurosci. 2016;19(6):835-844.

20. Wang H, et al. Gonadotrope-specific deletion of Dicer results in severely suppressed gonadotropins and fertility defects. J Biol Chem. 2015;290(5):2699-2714.

21. Hasuwa H, Ueda J, Ikawa M, Okabe M. miR$200 \mathrm{~b}$ and miR-429 function in mouse ovulation and are essential for female fertility. Science. 2013;341(6141):71-73.

22. Latreille $\mathrm{M}$, et al. MicroRNA-7a regulates pancreatic $\beta$ cell function. J Clin Invest. 2014;124(6):2722-2735.

23. Choudhury NR, et al. Tissue-specific control of brain-enriched miR-7 biogenesis. Genes Dev. 2013;27(1):24-38.

24. Memczak S, et al. Circular RNAs are a large class of animal RNAs with regulatory potency. Nature. 2013;495(7441):333-338.

25. Hansen TB, et al. Natural RNA circles function as efficient microRNA sponges. Nature. 2013;495(7441):384-388.

26. Horsham JL, Ganda C, Kalinowski FC, Brown RA, Epis MR, Leedman PJ. MicroRNA-7: A miRNA with expanding roles in development and disease. Int J Biochem Cell Biol. 2015;69:215-224.

27. Gu DN, Huang Q, Tian L. The molecular mechanisms and therapeutic potential of microRNA-7 in cancer. Expert Opin Ther Targets. 2015;19(3):415-426.

28. Junn E, Lee KW, Jeong BS, Chan TW, Im JY, Mouradian MM. Repression of alpha-synuclein expression and toxicity by microRNA-7. Proc Natl Acad Sci USA. 2009;106(31):13052-13057.

29. Amador A, Parkening T, Beamer W, Bartke A, Collins TJ. Autoregulation of testicular luteinizing hormone receptors in hypogonadal (hpg/ hpg) mice. Biochem Genet. 1984;22(5-6):395-401.

30. Good DJ, Porter FD, Mahon KA, Parlow AF, Westphal H, Kirsch IR. Hypogonadism and obesity in mice with a targeted deletion of the Nhlh2 gene. Nat Genet. 1997;15(4):397-401.

31. Eldar-Geva T, Hirsch HJ, Benarroch F, Rubinstein $\mathrm{O}$, Gross-Tsur V. Hypogonadism in females with Prader-Willi syndrome from infancy to adulthood: variable combinations of a primary gonadal defect and hypothalamic dysfunction. Eur $J$ Endocrinol. 2010;162(2):377-384.

32. Denzler R, Agarwal V, Stefano J, Bartel DP, Stoffel M. Assessing the ceRNA hypothesis with quantitative measurements of miRNA and target abundance. Mol Cell. 2014;54(5):766-776.

33. Horseman ND, et al. Defective mammopoiesis, but normal hematopoiesis, in mice with a targeted disruption of the prolactin gene. $E M B O \mathrm{~J}$. 1997;16(23):6926-6935.
34. Navarro VM, et al. Developmental and hormonally regulated messenger ribonucleic acid expression of KiSS-1 and its putative receptor, GPR54, in rat hypothalamus and potent luteinizing hormone-releasing activity of KiSS-1 peptide. Endocrinology. 2004;145(10):4565-4574.

35. Smith JT, Cunningham MJ, Rissman EF, Clifton DK, Steiner RA. Regulation of Kiss1 gene expression in the brain of the female mouse. Endocrinology. 2005;146(9):3686-3692.

36. Ruzankina Y, et al. Deletion of the developmentally essential gene ATR in adult mice leads to age-related phenotypes and stem cell loss. Cell Stem Cell. 2007;1(1):113-126.

37. Michos O, et al. Reduction of BMP4 activity by gremlin 1 enables ureteric bud outgrowth and GDNF/WNT11 feedback signalling during kidney branching morphogenesis. Development . 2007;134(13):2397-2405.

38. Orlicky DJ. Negative regulatory activity of a prostaglandin $\mathrm{F} 2$ alpha receptor associated protein (FPRP). Prostaglandins Leukot Essent Fatty Acids. 1996;54(4):247-259.

39. Orlicky DJ, Lieber JG, Morin CL, Evans RM. Synthesis and accumulation of a receptor regulatory protein associated with lipid droplet accumulation in 3T3-L1 cells. J Lipid Res. 1998;39(6):1152-1161.

40. Ahn J, Febbraio M, Silverstein RL. A novel isoform of human Golgi complex-localized glycoprotein-1 (also known as E-selectin ligand-1, MG-160 and cysteine-rich fibroblast growth factor receptor) targets differential subcellular localization. J Cell Sci. 2005;118(Pt 8):1725-1731.

41. Exton JH. Regulation of phosphoinositide phospholipases by hormones, neurotransmitters, and other agonists linked to $\mathrm{G}$ proteins. Annu Rev Pharmacol Toxicol. 1996;36:481-509.

42. Nicol L, Faure MO, McNeilly JR, Fontaine J, Taragnat C, McNeilly AS. Bone morphogenetic protein- 4 interacts with activin and $\mathrm{GnRH}$ to modulate gonadotrophin secretion in LbetaT2 gonadotrophs. JEndocrinol. 2008;196(3):497-507.

43. Faure MO, et al. BMP-4 inhibits folliclestimulating hormone secretion in ewe pituitary. JEndocrinol. 2005;186(1):109-121.

44. Grattan DR. 60 Years of neuroendocrinology: The hypothalamo-prolactin axis. JEndocrinol. 2015;226(2):T101-T122.

45. Tchernof A, Després JP. Sex steroid hormones, sex hormone-binding globulin, and obesity in men and women. Horm Metab Res. 2000;32(11-12):526-536.

46. Sanek NA, Young WS. Investigating the in vivo expression patterns of miR-7 microRNA family members in the adult mouse brain. Microrna. 2012;1(1):11-18.

47. Kim SH. Congenital Hypogonadotropic Hypogonadism and Kallmann Syndrome: Past, Present, and Future. Endocrinol Metab (Seoul). 2015;30(4):456-466.

48. Young LS, Speight A, Charlton HM, Clayton 
RN. Pituitary gonadotropin-releasing hormone receptor regulation in the hypogonadotrophic hypogonadal (hpg) mouse. Endocrinology. 1983;113(1):55-61.

49. Behrman HR. Prostaglandins in hypothalamopituitary and ovarian function. Annu Rev Physiol. 1979;41:685-700.

50. Labhsetwar AP. Effects of prostaglandin F2-alpha on pituitary luteinizing hormone content of pregnant rats: a possible explanation for the luteolytic effect. J Reprod Fertil. 1970;23(1):155-159.

51. Labhsetwar AP. Do prostaglandins stimulate LH release and thereby cause luteolysis? Prostaglandins. 1973;3(5):729-732.

52. Ginther OJ, Gastal EL, Gastal MO, Beg MA. Effect of prostaglandin F2alpha on ovarian, adrenal, and pituitary hormones and on luteal blood flow in mares. Domest Anim Endocrinol. 2007;32(4):315-328.

53. Massagué J, Blain SW, Lo RS. TGFbeta signaling in growth control, cancer, and heritable disorders. Cell. 2000;103(2):295-309.

54. Paez-Pereda M, et al. Involvement of bone morphogenetic protein 4 (BMP-4) in pituitary prolactinoma pathogenesis through a Smad/ estrogen receptor crosstalk. Proc Natl Acad Sci USA. 2003;100(3):1034-1039.

55. Haavisto AM, Pettersson K, Bergendahl M, Perheentupa A, Roser JF, Huhtaniemi I. A supersensitive immunofluorometric assay for rat luteinizing hormone. Endocrinology. 1993;132(4):1687-1691.

56. van Casteren JI, Schoonen WG, Kloosterboer HJ. Development of time-resolved immunofluorometric assays for rat follicle-stimulating hormone and luteinizing hormone and application on sera of cycling rats. Biol Reprod. 2000;62(4):886-894.

57. Yang M, Crawley JN. Simple behavioral assessment of mouse olfaction. Curr Protoc Neurosci. 2009; Chapter 8:Unit 8.24.

58. Windle JJ, Weiner RI, Mellon PL. Cell lines of the pituitary gonadotrope lineage derived by targeted oncogenesis in transgenic mice. Mol Endocrinol. 1990;4(4):597-603.

59. Thomas P, Mellon PL, Turgeon J, Waring DW. The $\mathrm{L}$ beta $\mathrm{T} 2$ clonal gonadotrope: a model for single cell studies of endocrine cell secretion. Endocrinology. 1996;137(7):2979-2989.

60. Garcia DM, Baek D, Shin C, Bell GW, Grimson A, Bartel DP. Weak seed-pairing stability and high target-site abundance decrease the proficiency of lsy-6 and other microRNAs. Nat Struct Mol Biol. 2011;18(10):1139-1146. 